\title{
TITLE:
}

\section{Unified theory of exactly and quasiexactly solvable "discrete" quantum mechanics. I. Formalism}

$\operatorname{AUTHOR}(S)$ :

Odake, Satoru; Sasaki, Ryu

\section{CITATION:}

Odake, Satoru ...[et al]. Unified theory of exactly and quasiexactly solvable "discrete" quantum mechanics. I. Formalism. Journal of Mathematical Physics 2010, 51(8): 083502.

\section{ISSUE DATE:}

2010-08

URL:

http://hdl.handle.net/2433/131811

RIGHT:

(C) 2010 American Institute of Physics 


\title{
Unified theory of exactly and quasiexactly solvable "discrete" quantum mechanics. I. Formalism
}

\author{
Satoru Odake ${ }^{1}$ and Ryu Sasaki ${ }^{2, a)}$ \\ ${ }^{1}$ Department of Physics, Shinshu University, Matsumoto 390-8621, Japan \\ ${ }^{2}$ Yukawa Institute for Theoretical Physics, Kyoto University, Kyoto 606-8502, Japan
}

(Received 23 March 2009; accepted 3 June 2010; published online 3 August 2010)

\begin{abstract}
We present a simple recipe to construct exactly and quasiexactly solvable Hamiltonians in one-dimensional "discrete" quantum mechanics, in which the Schrödinger equation is a difference equation. It reproduces all the known ones whose eigenfunctions consist of the Askey scheme of hypergeometric orthogonal polynomials of a continuous or a discrete variable. The recipe also predicts several new ones. An essential role is played by the sinusoidal coordinate, which generates the closure relation and the Askey-Wilson algebra together with the Hamiltonian. The relationship between the closure relation and the Askey-Wilson algebra is clarified. (C) 2010 American Institute of Physics. [doi:10.1063/1.3458866]
\end{abstract}

\section{INTRODUCTION}

For one-dimensional quantum mechanical systems, two sufficient conditions for exact solvability are known. The first is the shape invariance, ${ }^{1}$ which guarantees exact solvability in the Schödinger picture. The whole set of energy eigenvalues and the corresponding eigenfunctions can be obtained explicitly through shape invariance combined with Crum's theorem ${ }^{2}$ or the factorization method $^{3}$ or the supersymmetric quantum mechanics. ${ }^{4}$ The second is the closure relation. ${ }^{5}$ It allows to construct the exact Heisenberg operator solution of the sinusoidal coordinate $\eta(x)$, which generates the closure relation together with the Hamiltonian. The positive/negative energy parts of the Heisenberg operator solution give the annihilation/creation operators, in terms of which every eigenstate can be built up algebraically starting from the ground state. Thus, exact solvability in the Heisenberg picture is realized.

It is interesting to note that these two sufficient conditions apply equally well in the "discrete" quantum mechanics $(\mathrm{QM}),{ }^{6,7,5,8,9}$ which is a simple extension or deformation of QM. In discrete QM the dynamical variables are, as in the ordinary QM, the coordinate $x$ and the conjugate momentum $p$, which is realized as $p=-i \partial_{x}$. The Hamiltonian contains the momentum operator in exponentiated forms $e^{ \pm \beta p}$, which acts on wave functions as finite shift operators, either in the pure imaginary directions or the real directions. Thus, the Schrödinger equation in discrete QM is a difference equation instead of differential in ordinary QM. Various examples of exactly solvable discrete quantum mechanics are known for both of the two types of shifts, ${ }^{6,710,5,8,9}$ and the eigenfunctions consist of the Askey-scheme of the hypergeometric orthogonal polynomials ${ }^{11-13}$ of a continuous (pure imaginary shifts) and a discrete (real shifts) variable.

It should be stressed, however, that these two sufficient conditions do not tell how to build exactly solvable models. In this paper we present a simple theory of constructing exactly solvable Hamiltonians in discrete QM. It covers all the known examples of exactly solvable discrete QM with both pure imaginary and real shifts ${ }^{8,9}$ and it predicts several new ones to be explored in a subsequent publication. ${ }^{14}$ Moreover, the theory is general enough to generate quasiexactly solvable Hamiltonians in the same manner. The quasiexact solvability (QES) means, in contrast to the exact solvability, that only a finite number of energy eigenvalues and the corresponding eigen-

${ }^{a)}$ Electronic mail: ryu@yukawa.kyoto-u.ac.jp. 
functions can be obtained exactly. ${ }^{15}$ This unified theory also incorporates the known examples of quasiexactly solvable Hamiltonians. ${ }^{16,17}$ A new type of quasiexactly solvable Hamiltonians is constructed in this paper and its explicit examples will be surveyed in a subsequent publication. ${ }^{14}$ One of the merits of the present approach is that it reveals the common structure underlying the exactly and quasiexactly solvable theories. In ordinary QM, the corresponding theory was already given in Appendix A of Ref. 5, although it does not cover the quasiexact solvability.

The present paper is organized as follows. In Sec. II the general setting of the discrete quantum mechanics is briefly reviewed, and in Sec. II A the Hamiltonians for the pure imaginary shifts and for the real shifts cases are given and the general strategy of working in the vector space of polynomials in the sinusoidal coordinate is explained. In Sec. II B, based on a few postulates, various properties of the sinusoidal coordinate $\eta(x)$, which is the essential ingredient of the present theory, are presented in some detail. The main result of the paper, the unified form of the exactly and quasiexactly solvable "Hamiltonians," is given in Sec. II C. The action of the Hamiltonian on the polynomials of the sinusoidal coordinate is explained in Sec. II D. It simply maps a degree $n$ polynomial into a degree $n+L-2$ polynomial. Here $L$ is the degree of a certain polynomial constituting the potential function in the Hamiltonian. The exactly solvable case $(L=2)$ is discussed in section three. In Sec. III A, the closure relation, which used to be verified for each given Hamiltonian, is shown to be satisfied once and for all by the proposed exactly solvable Hamiltonian. The nature of the dual-closure relation, which plays an important role in the theory of discrete QM with real shifts and the corresponding theory of orthogonal polynomials of a discrete variable, is examined and compared with that of the closure relation in Sec. III B. The relationship between the closure plus dual-closure relations and the Askey-Wilson algebra ${ }^{18-21}$ is elucidated in Sec. III C. In Sec. III D, shape invariance is explained and shown to be satisfied for the pure imaginary shifts case (Sec. III D 1) and for the real shifts case (Sec. III D 2). The quasiexactly solvable "Hamiltonians" are discussed in Sec. IV. The QES case with $L=3$ is achieved in Sec. IV A by adjusting the compensation term which is linear in $\eta(x)$. A new type of QES with $L=4$ is introduced in Sec. IV B, which has quadratic in $\eta(x)$ compensation terms. It is shown that QES is not possible for $L \geq 5$ in Sec. IV C. The issue of returning from the "Hamiltonian" in the polynomial space to the original Hamiltonian $\mathcal{H}$ is discussed in Sec. V. This is related to the properties of the (pseudo-)ground state $\phi_{0}$. Section VI is for a summary, containing the simple recipe to construct exactly and quasiexactly solvable Hamiltonians. Appendix A provides the explicit forms of the sinusoidal coordinates with which the actual exactly and quasiexactly solvable Hamiltonians are constructed. There are eight different $\eta(x)$ for the continuous variable $x$ and five for the discrete $x$. Appendix B gives the proof of the Hermiticity of the Hamiltonian, which is slightly more involved than in the ordinary QM. Appendix $\mathrm{C}$ recapitulates the elementary formulas for the eigenvalues and eigenvectors of an upper-triangular matrix, to which the exactly solvable $(L=2)$ "Hamiltonian" in the polynomial space reduces.

\section{II. “DISCRETE” QUANTUM MECHANICS}

Throughout this paper we consider "discrete" quantum mechanics of one degree of freedom. Discrete quantum mechanics is a generalization of quantum mechanics in which the Schödinger equation is a difference equation instead of differential in ordinary QM. ${ }^{6,7,10,5,8,9}$ In other words, the Hamiltonian contains the momentum operator $p=-i \partial_{x}$ in exponentiated forms $e^{ \pm \beta p}$ which work as shift operators on the wave function,

$$
e^{ \pm \beta p} \psi(x)=\psi(x \mp i \beta) .
$$

According to the two choices of the parameter $\beta$, either real or pure imaginary, we have two types of discrete QM; with (i) pure imaginary shifts or (ii) real shifts, respectively. In the case of pure imaginary shifts, $\psi(x \mp i \gamma), \gamma \in \mathbb{R}_{\neq 0}$, we require the wave function to be an analytic function of $x$ with its domain including the real axis or a part of it on which the dynamical variable $x$ is defined. For the real shift case, the difference equation gives constraints on wave functions only on equally 
spaced lattice points. Then we choose, after proper rescaling, the variable $x$ to be an integer, with the total number either finite $(N+1)$ or infinite.

To sum up, the dynamical variable $x$ of the one-dimensional discrete quantum mechanics takes continuous or discrete values,

$$
\begin{aligned}
& \text { imaginary shifts: } x \in \mathbb{R}, \quad x \in\left(x_{1}, x_{2}\right), \\
& \text { real shifts: } x \in \mathbb{Z}, \quad x \in[0, N] \text { or }[0, \infty) .
\end{aligned}
$$

Here $x_{1}, x_{2}$ may be finite, $-\infty$ or $+\infty$. Correspondingly, the inner product of the wave functions has the following form:

$$
\begin{aligned}
& \text { imaginary shifts: }(f, g)=\int_{x_{1}}^{x_{2}} f^{*}(x) g(x) d x, \\
& \text { real shifts: }(f, g)=\sum_{x=0}^{N} f(x)^{*} g(x) \text { or } \sum_{x=0}^{\infty} f(x)^{*} g(x),
\end{aligned}
$$

and the norm of $f(x)$ is $\|f\|=\sqrt{(f, f)}$. In the case of imaginary shifts, other functions appearing in the Hamiltonian need to be analytic in $x$ within the same domain. Let us introduce the $*$-operation on an analytic function, $*: f \mapsto f^{*}$. If $f(x)=\Sigma_{n} a_{n} x^{n}, a_{n} \in \mathrm{C}$, then $f^{*}(x)=\Sigma_{n} a_{n}^{*} x^{n}$, in which $a_{n}^{*}$ is the complex conjugation of $a_{n}$. Obviously $f^{* *}(x)=f(x)$ and $f(x)^{*}=f^{*}\left(x^{*}\right)$. If $f$ is an analytic function, so def

is $g(x)=f(x-a), a \in \mathrm{C}$. The $*$-operation on this analytic function is $g^{*}(x)=\left(f\left(x^{*}-a\right)\right)^{*}=f^{*}\left(x-a^{*}\right)$. If a function satisfies $f^{*}=f$, then it takes real values on the real line. The "absolute value" of an analytic function to be used in this paper is defined by $|f(x)|=\sqrt{f(x) f^{*}(x)}$, which is again analytic and real non-negative on the real axis. Note that the $*$-operation is used in the inner product for pure imaginary shifts case (2.4) so that the entire integrand is an analytic function, too. This is essential for the proof of Hermiticity to be presented in Appendix B.

In quantum mechanics, the eigenvalue problem of a given Hamiltonian is the central issue. In this paper, we will consider the Hamiltonians having finite or semi-infinite discrete energy levels only,

$$
0=\mathcal{E}(0)<\mathcal{E}(1)<\mathcal{E}(2)<\cdots .
$$

Here we have chosen the additive constant of the Hamiltonian so that the ground state energy vanishes. In other words, the Hamiltonian is positive semidefinite. It is a well known theorem in linear algebra that any positive semidefinite Hermitian matrix can be factorized as a product of a certain matrix, say $\mathcal{A}$, and its Hermitian conjugate $\mathcal{A}^{\dagger}$. As we will see shortly, the Hamiltonians of discrete quantum mechanics have the same property, both with the imaginary and real shifts.

\section{A. Hamiltonian and strategy}

The Hamiltonian of one-dimensional discrete quantum mechanics has a simple form,

$$
\mathcal{H}^{\operatorname{def}}=\varepsilon\left(\sqrt{V_{+}(x)} e^{\beta p} \sqrt{V_{-}(x)}+\sqrt{V_{-}(x)} e^{-\beta p} \sqrt{V_{+}(x)}-V_{+}(x)-V_{-}(x)\right) .
$$

Corresponding to the imaginary/real shifts cases, the parameter $\beta$, the potential functions $V_{ \pm}(x)$, and a sign factor $\varepsilon$ are

$$
\text { imaginary shifts: } \beta=\gamma, \quad \varepsilon=1, \quad V_{+}(x)=V(x), \quad V_{-}(x)=V^{*}(x) \text {, }
$$




$$
\text { real shifts: } \beta=i, \quad \varepsilon=-1, \quad V_{+}(x)=B(x), \quad V_{-}(x)=D(x),
$$

with $\gamma \in \mathbb{R}_{\neq 0}$. The potential functions $B(x)$ and $D(x)$ are positive and vanish at boundaries,

$$
B(x)>0, \quad D(x)>0, \quad D(0)=0, \quad B(N)=0 \quad \text { for the finite case. }
$$

As mentioned above, $e^{ \pm \beta p}$ are shift operators $e^{ \pm \beta p} f(x)=f(x \mp i \beta)$, and the Schrödinger equation,

$$
\mathcal{H} \phi_{n}(x)=\mathcal{E}(n) \phi_{n}(x), \quad n=0,1,2, \ldots,
$$

is a difference equation. The Hermiticity of the Hamiltonian is manifest for the real shifts case because the Hamiltonian is a real symmetric matrix. For the imaginary shifts case, see Appendix B.

This positive semidefinite Hamiltonian (2.7) can be factorized,

$$
\mathcal{H}=\mathcal{A}^{\dagger} \mathcal{A} .
$$

Corresponding to the imaginary/real shifts cases, $\mathcal{A}$ and $\mathcal{A}^{\dagger}$ are

$$
\begin{array}{cl}
\mathcal{A}=i\left(e^{\gamma p / 2} \sqrt{V^{*}(x)}-e^{-\gamma p / 2} \sqrt{V(x)}\right), & \mathcal{A}^{\dagger}=-i\left(\sqrt{V(x)} e^{\gamma p / 2}-\sqrt{V^{*}(x)} e^{-\gamma p / 2}\right), \\
\mathcal{A}=\sqrt{B(x)}-e^{\partial} \sqrt{D(x)}, \quad & \mathcal{A}^{\dagger}=\sqrt{B(x)}-\sqrt{D(x)} e^{-\partial} .
\end{array}
$$

The ground state wave function $\phi_{0}(x)$ is determined as a zero mode of $\mathcal{A}$,

$$
\mathcal{A} \phi_{0}(x)=0 \text {. }
$$

The similarity transformed Hamiltonian $\tilde{\mathcal{H}}$ in terms of the ground state wave function $\phi_{0}$ has a much simpler form than the original Hamiltonian $\mathcal{H}$,

$$
\begin{aligned}
\widetilde{\mathcal{H}}^{\operatorname{def}} & =\phi_{0}(x)^{-1} \circ \mathcal{H} \circ \phi_{0}(x), \\
& =\varepsilon\left(V_{+}(x)\left(e^{\beta p}-1\right)+V_{-}(x)\left(e^{-\beta p}-1\right)\right) .
\end{aligned}
$$

In the second equation we have used (2.14).

In the following we will take $\widetilde{\mathcal{H}}$ instead of $\mathcal{H}$ as the starting point. That is, we reverse the argument and construct directly the "Hamiltonian" $\widetilde{\mathcal{H}}(2.16)$ based on a certain function $\eta(x)$ to be called the sinusoidal coordinate. The necessary properties of the sinusoidal coordinate will be introduced in Sec. II B. The general strategy is to construct the "Hamiltonian" $\widetilde{\mathcal{H}}$ in such a way that it maps a polynomial in $\eta(x)$ into another: $\widetilde{\mathcal{H}} \mathcal{V}_{n} \subseteq \mathcal{V}_{n+L-2} \subset \mathcal{V}_{\infty}$. Here $\mathcal{V}_{n}\left(n \in \mathbb{Z}_{\geq 0}\right)$ is defined by

$$
\mathcal{V}_{n}=\operatorname{Span}\left[1, \eta(x), \ldots, \eta(x)^{n}\right], \quad \mathcal{V}_{\infty}{ }^{\operatorname{def}}=\lim _{n \rightarrow \infty} \mathcal{V}_{n} .
$$

The goal is achieved by choosing very special forms of $V_{ \pm}(x)$ as given in (2.30) and (2.31), that is, $V(x)$ and $V^{*}(x)$ or $B(x)$ and $D(x)$ are polynomials of degree $L$ in the sinusoidal coordinate $\eta(x)$ and its shifts $\eta(x \mp i \beta)$ divided by special quadratic polynomials in them. This provides a unified theory of exactly solvable and quasiexactly solvable discrete QM. Exactly solvable QMs are realized by choosing $\tilde{\mathcal{H}}$ in such a way $(L=2)$ that $\tilde{\mathcal{H}} \mathcal{V}_{n} \subseteq \mathcal{V}_{n}$ is satisfied for all $n$. Then the existence of an eigenfunction, or to be more precise, a degree $n$ eigenpolynomial, of $\tilde{\mathcal{H}}$ is guaranteed for each integer $n$. On the other hand, quasiexact solvability is attained by adjusting the parameters of $\widetilde{\mathcal{H}}$ in such a way $(L=3,4)$ that $\widetilde{\mathcal{H}}^{\prime} \mathcal{V}_{M} \subseteq \mathcal{V}_{M}$ is realized for an integer $M$. Here $\widetilde{\mathcal{H}}^{\prime}$ is a modification of $\widetilde{\mathcal{H}}$ by the addition of the compensation terms. Then the "Hamiltonian" $\widetilde{\mathcal{H}}$ ' has an $M+1=\operatorname{dim}\left(\mathcal{V}_{M}\right)$-dimensional invariant space, providing $M+1$ eigenpolynomials of $\tilde{\mathcal{H}}^{\prime}$. After ob- 
taining such (quasi-)exactly solvable "Hamiltonian" $\widetilde{\mathcal{H}}\left(\widetilde{\mathcal{H}}^{\prime}\right)$, we have to find the (quasi-)ground state wave function $\phi_{0}$ in order to return to the true Hamiltonian $\mathcal{H}\left(\mathcal{H}^{\prime}\right)$ by $(2.15)$. It should be noted that the existence of such a (quasi-)ground state wave function is not guaranteed a priori since we have started with $\widetilde{\mathcal{H}}$ instead of $\mathcal{H}$. In the case of quasiexactly solvable QM, the positive semidefiniteness of Hamiltonian (2.6) is, in general, lost due to the inclusion of the compensation terms to $\tilde{\mathcal{H}}^{\prime}$.

\section{B. Sinusoidal coordinate}

Motivated by the study in Refs. 5, 8, and 9, let us define a sinusoidal coordinate $\eta(x)$ as a real [or "real" analytic $\eta^{*}(x)=\eta(x)$ in the case of pure imaginary shifts] function of $x$ satisfying the following symmetric shift-addition property:

$$
\eta(x-i \beta)+\eta(x+i \beta)=\left(2+r_{1}^{(1)}\right) \eta(x)+r_{-1}^{(2)} .
$$

Here $r_{1}^{(1)}$ and $r_{-1}^{(2)}$ are real parameters and we assume $r_{1}^{(1)}>-4$. These two, $r_{1}^{(1)}$ and $r_{-1}^{(2)}$, are fundamental parameters appearing in both exactly and quasiexactly solvable dynamical systems. For the exactly solvable systems, these two parameters also manifest themselves (3.4) in the closure relation, another characterization of exact solvability, to be discussed in Sec. III A. Since a polynomial in $\eta(x)$ is also a polynomial in $a \eta(x)+b$ ( $a, b$ : real constants), we impose two conditions

$$
\eta(0)=0 \text { and } \eta(x) \text { :monotone increasing function, }
$$

which are not essential for (quasi-)exact solvability but important for expressing various formulas in a unified way. Here we have assumed $0 \in\left[x_{1}, x_{2}\right]$. For the real shifts case, such $\eta(x)$ satisfying (2.18) and (2.19) can be classified into five types (A9)-(A13) (Ref. 8) and they also satisfy condition (2.20). We impose another condition, to be called the symmetric shift-multiplication property,

$$
\eta(x-i \beta) \eta(x+i \beta)=(\eta(x)-\eta(-i \beta))(\eta(x)-\eta(i \beta)),
$$

together with $\eta(x) \neq \eta(x-i \beta) \neq \eta(x+i \beta) \neq \eta(x)$.

Two conditions (2.18) and (2.20) imply that any symmetric polynomial in $\eta(x-i \beta)$ and $\eta(x$ $+i \beta)$ is expressed as a polynomial in $\eta(x)$. Especially we have $(n \geq-1)$,

$$
g_{n}(x)=\frac{\eta(x-i \beta)^{n+1}-\eta(x+i \beta)^{n+1}}{\eta(x-i \beta)-\eta(x+i \beta)}=\left(\begin{array}{c}
\text { a polynomial of } \\
\text { degree } n \text { in } \eta(x)
\end{array}\right)=\sum_{k=0}^{n} g_{n}^{(k)} \eta(x)^{n-k} .
$$

The coefficient $g_{n}^{(k)}$ is real because $g_{n}^{*}(x)=g_{n}(x)$. We set $g_{n}^{(k)}=0$ except for $0 \leq k \leq n$. Since $g_{n}(x)$ satisfies the following three term recurrence relation:

$$
g_{n+1}(x)=(\eta(x-i \beta)+\eta(x+i \beta)) g_{n}(x)-\eta(x-i \beta) \eta(x+i \beta) g_{n-1}(x) \quad(n \geq 0),
$$

we can write down $g_{n}^{(k)}$ explicitly. Especially $g_{n}^{(k)}$ for $k=0,1$ are

$$
\begin{aligned}
& g_{n}^{(0)}=[n+1], \\
& g_{n}^{(1)}= \begin{cases}\frac{1}{6} n(n+1)(2 n+1) r_{-1}^{(2)} & \text { for } r_{1}^{(1)}=0 \\
\frac{n[n+1]-(n+1)[n]}{r_{1}^{(1)}} r_{-1}^{(2)} & \text { for } r_{1}^{(1)} \neq 0 .\end{cases}
\end{aligned}
$$

Here we have defined $[n]$ as 


$$
[n]= \begin{cases}n & \text { for } r_{1}^{(1)}=0 \\ \frac{e^{\alpha n}-e^{-\alpha n}}{e^{\alpha}-e^{-\alpha}} & \text { for } r_{1}^{(1)}>0 \quad\left(\Leftarrow r_{1}^{(1)}=\left(e^{\alpha / 2}-e^{-\alpha / 2}\right)^{2} \quad(\alpha>0)\right) \\ \frac{e^{i \alpha n}-e^{-i \alpha n}}{e^{i \alpha}-e^{-i \alpha}} & \text { for }-4<r_{1}^{(1)}<0 \quad\left(\Leftarrow r_{1}^{(1)}=\left(e^{i(\alpha / 2)}-e^{-i(\alpha / 2)}\right)^{2}(0<\alpha<\pi)\right) .\end{cases}
$$

Note that $r_{1}^{(1)}$ and $r_{-1}^{(2)}$ are expressed as

$$
r_{1}^{(1)}=[2]-2, \quad r_{-1}^{(2)}=\eta(-i \beta)+\eta(i \beta) .
$$

For $n, m \in \mathbb{Z}, n \geq m-1$, we have

$$
\sum_{r=m}^{n} g_{r}^{(1)}=\left\{\begin{array}{cl}
\frac{1}{12}(n+m+1)(n-m+1)\left(n^{2}+2 n+m^{2}\right) r_{-1}^{(2)} & \text { for } r_{1}^{(1)}=0 \\
\frac{(n+1)[n+1]-m[m]-\left[\frac{1}{2}\right]^{-2}\left[\frac{n+m+1}{2}\right]\left[\frac{n-m+1}{2}\right]}{r_{1}^{(1)}} r_{-1}^{(2)} & \text { for } r_{1}^{(1)} \neq 0 .
\end{array}\right.
$$

The following properties of $[n]$ are useful:

$$
\begin{gathered}
{[a][a+c]-[b][b+c]=[a-b][a+b+c],} \\
\sum_{r=m}^{n}[r]=\frac{\left[\frac{n+m}{2}\right]\left[\frac{n-m+1}{2}\right]}{\left[\frac{1}{2}\right]} \quad(n, m \in \mathbb{Z}, n \geq m-1) .
\end{gathered}
$$

\section{Potential functions}

The first goal is to construct a general form of the "Hamiltonian" $\tilde{\mathcal{H}}$, such that a polynomial in $\eta(x)$ is mapped into another. It is achieved by the following form of the potential functions $V_{ \pm}(x)$ :

$$
\begin{aligned}
& V_{ \pm}(x)=\frac{\tilde{V}_{ \pm}(x)}{(\eta(x \mp i \beta)-\eta(x))(\eta(x \mp i \beta)-\eta(x \pm i \beta))}, \\
& \tilde{V}_{ \pm}(x)=\sum_{\substack{k, l \geq 0 \\
k+l \leq L}} v_{k, l} \eta(x)^{k} \eta(x \mp i \beta)^{l},
\end{aligned}
$$

where $L$ is a natural number roughly indicating the degree of $\eta(x)$ in $\tilde{V}_{ \pm}(x)$ and $v_{k, l}$ are real constants, with the constraint $\Sigma_{k+l=L} v_{k, l}^{2} \neq 0$. It is important that the same $v_{k, l}$ appears in both $\widetilde{V}_{ \pm}(x)$. As we will see in Sec. II D, the "Hamiltonian" $\widetilde{\mathcal{H}}$ with the above $V_{ \pm}(x)$ maps a degree $n$ polynomial in $\eta(x)$ to a degree $n+L-2$ polynomial (2.36) and (2.39).

The essential part of formula (2.30) is the denominators. They have the same form as the generic formula, derived by the present authors, for the coefficients of the three term recurrence relations of the orthogonal polynomials, (4.52) and (4.53) in Ref. 8. The translation rules are the duality correspondence itself, (3.14)-(3.18) in Ref. 8, 


$$
\begin{gathered}
\mathcal{E}(n) \rightarrow \eta(x), \quad-A_{n} \rightarrow V_{+}(x), \quad-C_{n} \rightarrow V_{-}(x), \\
\alpha_{+}(\mathcal{E}(n)) \rightarrow \eta(x-i \beta)-\eta(x), \quad \alpha_{-}(\mathcal{E}(n)) \rightarrow \eta(x+i \beta)-\eta(x) .
\end{gathered}
$$

Some of the parameters $v_{k, l}$ in (2.31) are redundant. From (2.18) and (2.20), we have

$$
\eta(x \mp i \beta)^{2}=\left(2+r_{1}^{(1)}\right) \eta(x) \eta(x \mp i \beta)-\eta(x)^{2}+r_{-1}^{(2)}(\eta(x)+\eta(x \mp i \beta))-\eta(-i \beta) \eta(i \beta) .
$$

By using this repeatedly, a monomial $\eta(x \mp i \beta)^{l}$ can be reduced to a polynomial of degree one in $\eta(x \mp i \beta)$ whose coefficients are polynomials in $\eta(x)$. Therefore, it is sufficient to keep $v_{k, l}$ with $l=0,1$. The remaining $2 L+1$ parameters $v_{k, l}(k+l \leq L, l=0,1)$ are independent, with one of which corresponds to the overall normalization of the Hamiltonian. In fact, if two sets of parameters $\left\{v_{k, l}\right\}$ and $\left\{v_{k, l}^{\prime}\right\} \quad(k+l \leq L, l=0,1)$ give the same $V_{ \pm}(x)$, namely, $\Sigma_{k=0}^{L}\left(v_{k, 0}-v_{k, 0}^{\prime}\right) \eta(x)^{k}+\Sigma_{k=0}^{L-1}\left(v_{k, 1}\right.$ $\left.-v_{k, 1}^{\prime}\right) \eta(x)^{k} \eta(x \mp i \beta)=0$, then we obtain $v_{k, l}=v_{k, l}^{\prime}$. Therefore, there is no more redundancy in $v_{k, l}$ $(k+l \leq L, l=0,1)$. Note that we have not yet imposed the boundary condition $D(0)=0(2.9)$. The sinusoidal coordinate $\eta(x)$ itself may have extra parameters.

\section{D. $\tilde{\mathcal{H}}$ on the polynomial space}

The action of $\tilde{\mathcal{H}}(2.16)$ on $\eta(x)^{n}$ becomes with (2.30) and (2.31),

$$
\begin{aligned}
& \widetilde{\mathcal{H}} \eta(x)^{n}=\varepsilon\left(V_{+}(x)\left(\eta(x-i \beta)^{n}-\eta(x)^{n}\right)+V_{-}(x)\left(\eta(x+i \beta)^{n}-\eta(x)^{n}\right)\right) \\
& =\varepsilon \frac{\tilde{V}_{+}(x) \sum_{r=0}^{n-1} \eta(x)^{r} \eta(x-i \beta)^{n-1-r}-\tilde{V}_{-}(x) \sum_{r=0}^{n-1} \eta(x)^{r} \eta(x+i \beta)^{n-1-r}}{\eta(x-i \beta)-\eta(x+i \beta)} \\
& =\varepsilon \sum_{r=0}^{n-1} \eta(x)^{r} \frac{\tilde{V}_{+}(x) \eta(x-i \beta)^{n-1-r}-\tilde{V}_{-}(x) \eta(x+i \beta)^{n-1-r}}{\eta(x-i \beta)-\eta(x+i \beta)} \\
& =\varepsilon \sum_{r=0}^{n-1} \eta(x)^{r} \sum_{\substack{k, l \geq 0 \\
k+l \leq L}} v_{k, l} \eta(x)^{k} \frac{\eta(x-i \beta)^{l+n-1-r}-\eta(x+i \beta)^{l+n-1-r}}{\eta(x-i \beta)-\eta(x+i \beta)} \\
& =\varepsilon \sum_{k, l \geq 0} v_{k, l} \sum_{r=0}^{n-1} \eta(x)^{k+r} g_{n+l-r-2}(x)=(\text { a polynomial of degree } n+L-2 \text { in } \eta(x)) \\
& k+l \leq L \\
& =\varepsilon \sum_{k, l \geq 0} \sum_{r=0}^{n-1} \sum_{j=0}^{n+l-r-2} v_{k, l} g_{n+l-r-2}^{(j)} \eta(x)^{n+k+l-2-j} \\
& k+l \leq L \\
& =\varepsilon \sum_{m=0}^{n+L-2} \eta(x)^{n+L-2-m} \sum_{j=\max (m-L, 0)}^{m} \sum_{\substack{k, l \geq 0 \\
k+l=L-m+j}} v_{k, l} \sum_{r=0}^{n-1} g_{n+l-r-2}^{(j)} \\
& =\sum_{m=0}^{n+L-2} \eta(x)^{n+L-2-m} \sum_{j=\max (m-L, 0)}^{m} e_{m, j, n} .
\end{aligned}
$$

Here $e_{m, j, n}$ (the $L$-dependence is implicit) is defined by 


$$
e_{m, j, n}=\varepsilon \sum_{l=0}^{\operatorname{def}} v_{L-m+j-l, l} \sum_{r=0}^{n-1} g_{n+l-r-2}^{(j)} \text {. }
$$

Therefore, the matrix elements of $\tilde{\mathcal{H}}$ in the basis $\left\{\eta(x)^{n}\right\}_{n=0,1, \ldots}$ is given by

$$
\tilde{\mathcal{H}} \eta(x)^{n}=\sum_{m=0}^{n+L-2} \eta(x)^{m} \tilde{\mathcal{H}}_{m, n}^{\eta}, \quad \tilde{\mathcal{H}}_{m, n}^{\eta}=\sum_{j=\max (n-2-m, 0)}^{n+L-2-m} e_{n+L-2-m, j, n} .
$$

The coefficients $e_{m, 0, n}$ and $e_{m, 1, n}$ become, by using (2.29) and (2.27),

$$
\begin{aligned}
& e_{m, 0, n}=\varepsilon \frac{\left[\frac{n}{2}\right]}{\left[\frac{1}{2}\right]} \sum_{l=0}^{L-m} v_{L-m-l, l}\left[\frac{n+2 l-1}{2}\right], \\
& e_{m, 1, n}=\varepsilon \sum_{l=0}^{L-m+1} v_{L-m+1-l, l} \\
& \times \begin{cases}\frac{1}{12} n(n+2 l-2)\left((n+l-1)^{2}+l^{2}-2 l\right) r_{-1}^{(2)} & \text { for } r_{1}^{(1)}=0 \\
\frac{(n+l-1)[n+l-1]-(l-1)[l-1]-\left[\frac{1}{2}\right]^{-2}\left[\frac{n+2 l-2}{2}\right]\left[\frac{n}{2}\right]}{r_{1}^{(1)}} r_{-1}^{(2)} & \text { for } r_{1}^{(1)} \neq 0 .\end{cases}
\end{aligned}
$$

So far the conditions $v_{k, l}=0$ for $l \geq 2$ are not used.

We have established

$$
\tilde{\mathcal{H}} \mathcal{V}_{n} \subseteq \mathcal{V}_{n+L-2}
$$

where $\mathcal{V}_{n}$ is the polynomial space defined in (2.17). For $L=2, \mathcal{V}_{n}$ is $\tilde{\mathcal{H}}$-invariant. Therefore, this case is exactly solvable; all the eigenvalues and eigenfunctions of $\tilde{\mathcal{H}}$ can be obtained explicitly and the eigenfunction is a polynomial of degree $n$ in $\eta(x)$ for each $n$. On the other hand, $L \geq 3$ cases are not exactly solvable but some cases can be made quasiexactly solvable by certain modification to be discussed presently. For $L=0,1$ cases, the matrix $\tilde{\mathcal{H}}^{\eta}=\left(\widetilde{\mathcal{H}}_{m, n}^{\eta}\right)_{0 \leq m, n \leq K}$ with finite $K$ is not diagonalizable except for $K=0,1$.

In the following we will set $v_{k, l}=0$ for $l \geq 2$, see Sec. II C. For the real shifts case, the condition $D(0)=0(2.9)$ is satisfied by choosing $v_{0,0}$ as $v_{0,0}=-v_{0,1} \eta(-1)$.

\section{EXACTLY SOLVABLE $\tilde{\mathcal{H}}$}

The $L=2$ case is exactly solvable. Since the Hamiltonian of the polynomial space $\tilde{\mathcal{H}}$ is an upper-triangular matrix (2.36), its eigenvalues and eigenvectors are easily obtained explicitly, see Appendix C. The eigenvalue $\mathcal{E}(n)$ is 


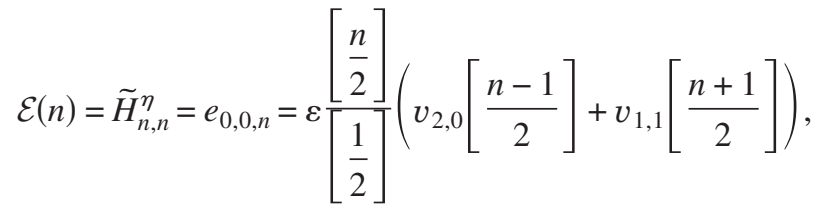

and the corresponding eigenpolynomial $P_{n}(\eta(x))$ is expressed as a determinant of the following order $n+1$ matrix:

$$
P_{n}(\eta(x)) \propto\left|\begin{array}{ccccc}
1 & \eta(x) & \eta(x)^{2} & \ldots & \eta(x)^{n} \\
\mathcal{E}(0)-\mathcal{E}(n) & \widetilde{H}_{0,1}^{\eta} & \widetilde{H}_{0,2}^{\eta} & \ldots & \widetilde{H}_{0, n}^{\eta} \\
& \mathcal{E}(1)-\mathcal{E}(n) & \widetilde{H}_{1,2}^{\eta} & \ldots & \widetilde{H}_{1, n}^{\eta} \\
& & \ddots & \ddots & \vdots \\
0 & & & \mathcal{E}(n-1)-\mathcal{E}(n) & \widetilde{H}_{n-1, n}^{\eta}
\end{array}\right| .
$$

For a choice of the sinusoidal coordinate among the possible forms (A1)-(A13) and the values of the five parameters, $v_{0,0}, v_{1,0}, v_{0,1}, v_{1,1}$, and $v_{2,0}$, these two formulas (3.1) and (3.2), although clumsy, give the complete solutions of the "Schrödinger equation" $\tilde{\mathcal{H}} P_{n}(\eta(x))=\mathcal{E}(n) P_{n}(\eta(x))$ at the algebraic level. For the solutions of a full quantum mechanical problem, however, one needs the square-integrable ground state wave function $\phi_{0}(x)$ (2.14), which is essential for the existence of the Hamiltonian $\mathcal{H}$ and the verification of its Hermiticity. These conditions would usually restrict the ranges of the parameters $v_{0,0}, \ldots, v_{2,0}$.

For specific problems, however, there are more powerful and systematic solution methods based on the shape invariance ${ }^{1,6-9}$ and the closure relation. ${ }^{5,8,9}$ These two are independent and sufficient conditions for exact solvability which are applicable to not only ordinary QM but also discrete QM. In our previous works ${ }^{6,7,8,9}$ these conditions were verified for each specific problem. Here we will provide proofs based on the generic form of the exactly solvable $(L=2)$ "Hamiltonian" $\widetilde{\mathcal{H}},(2.16),(2.30)$, and (2.31). These proofs apply to all the exactly solvable discrete QM. In the rest of this section we assume the existence of the ground state wave function $\phi_{0}(x)(2.14)$.

\section{A. Closure relation}

The closure relation is a commutator relation between the Hamiltonian $\mathcal{H}$ and the sinusoidal coordinate $\eta(x),{ }^{5,8,9}$

$$
[\mathcal{H},[\mathcal{H}, \eta]]=\eta R_{0}(\mathcal{H})+[\mathcal{H}, \eta] R_{1}(\mathcal{H})+R_{-1}(\mathcal{H}) .
$$

Here $R_{i}(z)$ are polynomials with real coefficients $r_{i}^{(j)}$,

$$
R_{1}(z)=r_{1}^{(1)} z+r_{1}^{(0)}, \quad R_{0}(z)=r_{0}^{(2)} z^{2}+r_{0}^{(1)} z+r_{0}^{(0)}, \quad R_{-1}(z)=r_{-1}^{(2)} z^{2}+r_{-1}^{(1)} z+r_{-1}^{(0)} .
$$

Reflecting the fact that the Hamiltonian $\mathcal{H}$ has shift operators $e^{ \pm \beta p}$, whereas $\eta(x)$ has none, the functions $R_{0}(\mathcal{H})$ and $R_{-1}(\mathcal{H})$ are quadratic in $\mathcal{H}$ and $R_{1}(\mathcal{H})$ is linear in $\mathcal{H}$. By similarity transforming (3.3) in terms of the ground state wave function $\phi_{0}$, it is rewritten as

$$
[\tilde{\mathcal{H}},[\tilde{\mathcal{H}}, \eta]]=\eta R_{0}(\tilde{\mathcal{H}})+[\tilde{\mathcal{H}}, \eta] R_{1}(\tilde{\mathcal{H}})+R_{-1}(\tilde{\mathcal{H}}) .
$$

Closure relation (3.3) allows us to obtain the exact Heisenberg operator solution for $\eta(x)$, and the annihilation and creation operators $a^{( \pm)}$are extracted from this exact Heisenberg operator solution, ${ }^{5}$

$$
e^{i t \mathcal{H}} \eta(x) e^{-i t \mathcal{H}}=a^{(+)} e^{i \alpha_{+}(\mathcal{H}) t}+a^{(-)} e^{i \alpha_{-}(\mathcal{H}) t}-R_{-1}(\mathcal{H}) R_{0}(\mathcal{H})^{-1},
$$




$$
\begin{gathered}
\alpha_{ \pm}(\mathcal{H})=\frac{\operatorname{def}}{2}\left(R_{1}(\mathcal{H}) \pm \sqrt{R_{1}(\mathcal{H})^{2}+4 R_{0}(\mathcal{H})}\right) \\
R_{1}(\mathcal{H})=\alpha_{+}(\mathcal{H})+\alpha_{-}(\mathcal{H}), \quad R_{0}(\mathcal{H})=-\alpha_{+}(\mathcal{H}) \alpha_{-}(\mathcal{H}) \\
a^{( \pm)}= \pm\left([\mathcal{H}, \eta(x)]-\left(\eta(x)+R_{-1}(\mathcal{H}) R_{0}(\mathcal{H})^{-1}\right) \alpha_{\mp}(\mathcal{H})\right)\left(\alpha_{+}(\mathcal{H})-\alpha_{-}(\mathcal{H})\right)^{-1} \\
= \pm\left(\alpha_{+}(\mathcal{H})-\alpha_{-}(\mathcal{H})\right)^{-1}\left([\mathcal{H}, \eta(x)]+\alpha_{ \pm}(\mathcal{H})\left(\eta(x)+R_{-1}(\mathcal{H}) R_{0}(\mathcal{H})^{-1}\right)\right)
\end{gathered}
$$

The energy spectrum is determined by the overdetermined recursion relations $\mathcal{E}(n+1)=\mathcal{E}(n)$ $+\alpha_{+}(\mathcal{E}(n))$ and $\mathcal{E}(n-1)=\mathcal{E}(n)+\alpha_{-}(\mathcal{E}(n))$ with $\mathcal{E}(0)=0$, and the excited state wave functions $\left\{\phi_{n}(x)\right\}$ are obtained by successive action of the creation operator $a^{(+)}$on the ground state wave function $\phi_{0}(x)$. Closure relation (3.5) [or (3.3)] is equivalent to the following set of five equations:

$$
\begin{gathered}
\eta(x-2 i \beta)-2 \eta(x-i \beta)+\eta(x)=r_{0}^{(2)} \eta(x)+r_{-1}^{(2)}+r_{1}^{(1)}(\eta(x-i \beta)-\eta(x)) \\
\eta(x+2 i \beta)-2 \eta(x+i \beta)+\eta(x)=r_{0}^{(2)} \eta(x)+r_{-1}^{(2)}+r_{1}^{(1)}(\eta(x+i \beta)-\eta(x)) \\
(\eta(x-i \beta)-\eta(x))\left(V_{+}(x-i \beta)+V_{-}(x-i \beta)-V_{+}(x)-V_{-}(x)\right) \\
=-\left(r_{0}^{(2)} \eta(x)+r_{-1}^{(2)}\right)\left(V_{+}(x-i \beta)+V_{-}(x-i \beta)+V_{+}(x)+V_{-}(x)\right) \\
-r_{1}^{(1)}(\eta(x-i \beta)-\eta(x))\left(V_{+}(x-i \beta)+V_{-}(x-i \beta)\right) \\
+\varepsilon^{-1}\left(r_{0}^{(1)} \eta(x)+r_{-1}^{(1)}+r_{1}^{(0)}(\eta(x-i \beta)-\eta(x))\right) \\
(\eta(x+i \beta)-\eta(x))\left(V_{+}(x+i \beta)+V_{-}(x+i \beta)-V_{+}(x)-V_{-}(x)\right) \\
=-\left(r_{0}^{(2)} \eta(x)+r_{-1}^{(2)}\right)\left(V_{+}(x+i \beta)+V_{-}(x+i \beta)+V_{+}(x)+V_{-}(x)\right) \\
-r_{1}^{(1)}(\eta(x+i \beta)-\eta(x))\left(V_{+}(x+i \beta)+V_{-}(x+i \beta)\right) \\
+\varepsilon^{-1}\left(r_{0}^{(1)} \eta(x)+r_{-1}^{(1)}+r_{1}^{(0)}(\eta(x+i \beta)-\eta(x))\right) \\
2(\eta(x)-\eta(x-i \beta)) V_{+}(x) V_{-}(x-i \beta)+2(\eta(x)-\eta(x+i \beta)) V_{-}(x) V_{+}(x+i \beta) \\
=\left(r_{0}^{(2)} \eta(x)+r_{-1}^{(2)}\right)\left(V_{+}(x) V_{-}(x-i \beta)+V_{-}(x) V_{+}(x+i \beta)+\left(V_{+}(x)+V_{-}(x)\right)^{2}\right) \\
+r_{1}^{(1)}(\eta(x-i \beta)-\eta(x)) V_{+}(x) V_{-}(x-i \beta)+r_{1}^{(1)}(\eta(x+i \beta)-\eta(x)) V_{-}(x) V_{+}(x+i \beta) \\
-\varepsilon^{-1}\left(r_{0}^{(1)} \eta(x)+r_{-1}^{(1)}\right)\left(V_{+}(x)+V_{-}(x)\right)+\varepsilon^{-2}\left(r_{0}^{(0)} \eta(x)+r_{-1}^{(0)}\right)
\end{gathered}
$$

Obviously (3.11) and (3.12) are equivalent and so are (3.13) and (3.14), under the condition (3.16). By substituting our choice of $V_{ \pm}(x)(2.30)$ and (2.31) for $L=2$, it is straightforward to verify the other three equations (3.13)-(3.15). The coefficients $r_{i}^{(j)}$ appearing in (3.4) are expressed by the parameters $v_{1,0}, v_{0,1}, v_{1,1}$, and $v_{2,0}$ together with the two parameters $r_{1}^{(1)}$ and $r_{-1}^{(2)}$ which have already appeared in the definition of $\eta(x)(2.18)$ [see also (2.26)],

$$
\begin{gathered}
r_{0}^{(2)}=r_{1}^{(1)}, \quad r_{0}^{(1)}=2 r_{1}^{(0)}, \\
\varepsilon^{-1} r_{1}^{(0)}=v_{2,0}+v_{1,1}, \quad \varepsilon^{-2} r_{0}^{(0)}=-v_{2,0} v_{1,1},
\end{gathered}
$$




$$
\varepsilon^{-1} r_{-1}^{(1)}=v_{1,0}+v_{0,1}, \quad \varepsilon^{-2} r_{-1}^{(0)}=-v_{2,0} v_{0,1} .
$$

Note that $v_{0,0}$ does not appear. It implies that for the imaginary shifts case, the commutation relation between the annihilation and creation operators does not depend on $v_{0,0}$. With these formulas, the explicit forms of $\alpha_{ \pm}(\mathcal{H})$ (3.7) can be expressed in terms of $r_{1}^{(1)}, v_{2,0}$, and $v_{1,1}$. It is straightforward to verify eigenvalue formula (3.1). This concludes the unified proof of the closure relation for all the discrete $\mathrm{QM}$.

\section{B. Dual-closure relation}

The dual-closure relation has the same forms as closure relation (3.3) and (3.5) with the roles of Hamiltonian $\mathcal{H}(\tilde{\mathcal{H}})$ and the sinusoidal coordinate $\eta(x)$ exchanged,

$$
\begin{aligned}
& {[\eta,[\eta, \mathcal{H}]]=\mathcal{H} R_{0}^{\text {dual }}(\eta)+[\eta, \mathcal{H}] R_{1}^{\text {dual }}(\eta)+R_{-1}^{\text {dual }}(\eta),} \\
& {[\eta,[\eta, \widetilde{\mathcal{H}}]]=\widetilde{\mathcal{H}} R_{0}^{\text {dual }}(\eta)+[\eta, \widetilde{\mathcal{H}}] R_{1}^{\text {dual }}(\eta)+R_{-1}^{\text {dual }}(\eta),}
\end{aligned}
$$

where $R_{i}^{\text {dual }}(z)$ are as yet unknown polynomials. We will show below that the dual-closure relation is the characteristic feature shared by all the "Hamiltonians" $\widetilde{\mathcal{H}}$ which map a polynomial in $\eta(x)$ into another. Therefore, its dynamical contents are not so constraining as the closure relation, except for the real shifts (the discrete variable) exactly solvable $(L=2)$ case, where the closure relation and the dual-closure relations are on the same footing as shown in Ref. 8. By substituting the "Hamiltonian" $\tilde{\mathcal{H}}(2.16)$ without any further specification of $V_{ \pm}$into the above (3.20), we find it is equivalent to the following set of three equations:

$$
\begin{gathered}
(\eta(x)-\eta(x-i \beta))^{2}=R_{0}^{\mathrm{dual}}(\eta(x-i \beta))+(\eta(x)-\eta(x-i \beta)) R_{1}^{\mathrm{dual}}(\eta(x-i \beta)), \\
(\eta(x)-\eta(x+i \beta))^{2}=R_{0}^{\mathrm{dual}}(\eta(x+i \beta))+(\eta(x)-\eta(x+i \beta)) R_{1}^{\mathrm{dual}}(\eta(x+i \beta)), \\
0=-\varepsilon\left(V_{+}(x)+V_{-}(x)\right) R_{0}^{\mathrm{dual}}(\eta(x))+R_{-1}^{\mathrm{dual}}(\eta(x)) .
\end{gathered}
$$

These imply

$$
\begin{aligned}
& R_{1}^{\text {dual }}(\eta(x))=(\eta(x-i \beta)-\eta(x))+(\eta(x+i \beta)-\eta(x)), \\
& R_{0}^{\text {dual }}(\eta(x))=-(\eta(x-i \beta)-\eta(x))(\eta(x+i \beta)-\eta(x)), \\
& R_{-1}^{\text {dual }}(\eta(x))=\varepsilon\left(V_{+}(x)+V_{-}(x)\right) R_{0}^{\text {dual }}(\eta(x)) .
\end{aligned}
$$

By using the defining properties of sinusoidal coordinate (2.18)-(2.20), we actually find that $R_{1}^{\text {dual }}(z)$ is a degree 1 polynomial in $z$ and $R_{0}^{\text {dual }}(z)$ is a quadratic polynomial,

$$
\begin{aligned}
& R_{1}^{\text {dual }}(z)=r_{1}^{(1)} z+r_{-1}^{(2)}, \\
& R_{0}^{\text {dual }}(z)=r_{1}^{(1)} z^{2}+2 r_{-1}^{(2)} z-\eta(-i \beta) \eta(i \beta) .
\end{aligned}
$$

By using the explicit forms of $V_{ \pm}(2.30)$ and (2.31) (with an arbitrary $L$ ), we obtain

$$
R_{-1}^{\text {dual }}(z)=\varepsilon\left(v_{0,0}+\sum_{k=1}^{L}\left(v_{k, 0}+v_{k-1,1}\right) z^{k}\right) .
$$

Therefore, all $R_{i}^{\text {dual }}(z)$ are polynomials and the dual-closure relation is demonstrated in a unified fashion for an arbitrary $L$. Thus, it does not characterize the exact nor the quasiexact solvability. 
For the exactly solvable $L=2$ case, by using (3.17) and (3.18), $R_{-1}^{\text {dual }}(z)$ can be written as

$$
R_{-1}^{\text {dual }}(z)=r_{1}^{(0)} z^{2}+r_{-1}^{(1)} z+\varepsilon v_{0,0} .
$$

For the real shifts case, in order to satisfy $D(0)=0(2.9)$, we have to take $v_{0,0}=-\eta(-1) v_{0,1}$, and this implies $\varepsilon v_{0,0}=\eta(1) \eta(-1) B(0)$. See (4.104)-(4.106) in Ref. 8.

\section{Askey-Wilson algebra}

Here we will focus on the exactly solvable systems and will briefly comment on the relationship between the closure plus the dual-closure relations and the so-called Askey-Wilson algebra. ${ }^{18-21}$ By simply expanding the double commutators in closure (3.3) and dual-closure (3.19) relations, we obtain two cubic relations generated by the two operators $\mathcal{H}$ and $\eta$,

$$
\begin{aligned}
& \mathcal{H}^{2} \eta-\left(2+r_{1}^{(1)}\right) \mathcal{H} \eta \mathcal{H}+\eta \mathcal{H}^{2}-r_{1}^{(0)}(\mathcal{H} \eta+\eta \mathcal{H})-r_{0}^{(0)} \eta=r_{-1}^{(2)} \mathcal{H}^{2}+r_{-1}^{(1)} \mathcal{H}+r_{-1}^{(0)}, \\
& \eta^{2} \mathcal{H}-\left(2+r_{1}^{(1)}\right) \eta \mathcal{H} \eta+\mathcal{H} \eta^{2}-r_{-1}^{(2)}(\eta \mathcal{H}+\mathcal{H} \eta)+\eta(-i \beta) \eta(i \beta) \mathcal{H}=r_{1}^{(0)} \eta^{2}+r_{-1}^{(1)} \eta+\varepsilon v_{0,0} .
\end{aligned}
$$

From its structure, the closure relation is at most linear in $\eta$ and at most quadratic in $\mathcal{H}$. So the left hand side of (3.31) has terms containing one factor of $\eta$ and the right hand side, none. It is simply $R_{-1}(\mathcal{H})$. Likewise, the left hand side of (3.32) has terms containing one factor of $\mathcal{H}$ and the right hand side, none. It is simply $R_{-1}^{\text {dual }}(\mathcal{H})$. In $(3.32), \eta(-i \beta) \eta(i \beta)$ is just a real number, not an operator.

These have the same form as the so-called Askey-Wilson algebra, which has many different expressions. The original one is due to Zhedanov. ${ }^{18}$ Here we present a slightly more general version than the original one and is due to Refs. 19 and 20. It is generated by three elements $K_{1}$, $K_{2}, K_{3}$,

$$
\begin{aligned}
& {\left[K_{1}, K_{2}\right]=K_{3},} \\
& {\left[K_{3}, K_{1}\right]=2 \rho K_{1} K_{2} K_{1}+a_{2}\left(K_{1} K_{2}+K_{2} K_{1}\right)+a_{1} K_{1}^{2}+c_{2} K_{2}+d K_{1}+g_{2},} \\
& {\left[K_{2}, K_{3}\right]=2 \rho K_{2} K_{1} K_{2}+a_{1}\left(K_{2} K_{1}+K_{1} K_{2}\right)+a_{2} K_{2}^{2}+c_{1} K_{1}+d K_{2}+g_{1} .}
\end{aligned}
$$

By expanding the commutators and eliminating $K_{3}$, they are reduced to

$$
\begin{aligned}
& K_{1}^{2} K_{2}+2(\rho-1) K_{1} K_{2} K_{1}+K_{2} K_{1}^{2}+a_{2}\left(K_{1} K_{2}+K_{2} K_{1}\right)+c_{2} K_{2}=-a_{1} K_{1}^{2}-d K_{1}-g_{2}, \\
& K_{2}^{2} K_{1}+2(\rho-1) K_{2} K_{1} K_{2}+K_{1} K_{2}^{2}+a_{1}\left(K_{2} K_{1}+K_{1} K_{2}\right)+c_{1} K_{1}=-a_{2} K_{2}^{2}-d K_{2}-g_{1} .
\end{aligned}
$$

Another version due to Terwilliger ${ }^{21}$ is generated by two independent elements $A$ and $A^{\times}$and it has only expanded forms,

$$
\begin{gathered}
A^{2} A^{\times}-\beta_{\mathrm{T}} A A^{\times} A+A^{\times} A^{2}-\gamma\left(A A^{\times}+A^{\times} A\right)-\rho_{\mathrm{T}} A^{\times}=\gamma^{\times} A^{2}+\omega A+\eta_{\mathrm{T}}, \\
A^{\times 2} A-\beta_{\mathrm{T}} A^{\times} A A^{\times}+A A^{\times 2}-\gamma^{\times}\left(A^{\times} A+A A^{\times}\right)-\rho_{\mathrm{T}}^{\times} A=\gamma A^{\times 2}+\omega A^{\times}+\eta_{\mathrm{T}}^{\times} .
\end{gathered}
$$

Here is the list of correspondence of the generators and coefficients, 


$\begin{array}{ccc}\text { Refs. } 19 \text { and } 20 & \text { Ref. } 21 & \text { This paper } \\ K_{1} & A & \mathcal{H} \\ K_{2} & A^{\times} & \eta \\ 2(1-\rho) & \beta_{\mathrm{T}} & 2+r_{1}^{(1)} \\ -a_{2} & \gamma & r_{1}^{(0)}=\varepsilon\left(v_{2,0}+v_{1,1}\right) \\ -a_{1} & \gamma^{\times} & r_{-1}^{(2)}=\eta(-i \beta)+\eta(i \beta) \\ -c_{2} & \rho_{\mathrm{T}} & r_{0}^{(0)}=-\varepsilon^{2} v_{2,0} v_{1,1} \\ -c_{1} & \rho_{\mathrm{T}}^{\times} & -\eta(-i \beta) \eta(i \beta) \\ -d & \omega & r_{-1}^{(1)}=\varepsilon\left(v_{1,0}+v_{0,1}\right) \\ -g_{2} & \eta_{\mathrm{T}} & r_{-1}^{(0)}=-\varepsilon^{2} v_{2,0} v_{0,1} \\ -g_{1} & \eta_{\mathrm{T}}^{\times} & \varepsilon v_{0,0} .\end{array}$

In Ref. 19 the Casimir operator $Q$ commuting with all the generators of the algebra, $\left[K_{1}, Q\right]$ $=\left[K_{2}, Q\right]=\left[K_{3}, Q\right]=0$, is given as

$$
\begin{aligned}
Q= & K_{1} K_{2} K_{1} K_{2}+K_{2} K_{1} K_{2} K_{1}-(1-\rho)\left(K_{1} K_{2}^{2} K_{1}+K_{2} K_{1}^{2} K_{2}\right) \\
& +(2-\rho)\left(a_{1} K_{1} K_{2} K_{1}+a_{2} K_{2} K_{1} K_{2}\right)+(1-\rho)\left(c_{1} K_{1}^{2}+c_{2} K_{2}^{2}\right) \\
& +\left(d-a_{1} a_{2}\right)\left(K_{1} K_{2}+K_{2} K_{1}\right)+\left((2-\rho) g_{1}-a_{2} c_{1}\right) K_{1}+\left((2-\rho) g_{2}-a_{1} c_{2}\right) K_{2} .
\end{aligned}
$$

With the above substitution (3.40), $K_{1} \rightarrow \mathcal{H}, K_{2} \rightarrow \eta$, etc., the Casimir operator turns out to be a constant, ${ }^{22}$

$$
Q=\varepsilon^{2}\left(v_{1,1} v_{0,0}-v_{1,0} v_{0,1}-r_{-1}^{(2)} v_{2,0} v_{0,1}\right) .
$$

Although this fact might appear striking from the pure algebra point of view (3.33)-(3.35), it is rather trivial in quantum mechanics. In one-dimensional quantum mechanics, there is no dynamical operator which commutes with the Hamiltonian. Therefore, if $Q$ commutes with $\mathcal{H}$, it must be a constant.

Now here are some comments on the dissimilarity. The first obvious difference is the structure. While the Askey-Wilson algebra (3.33)-(3.35) or (3.38) and (3.39) has no inherent structure, closure relation (3.3) has the right structure to lead to the Heisenberg operator solution for $\eta(x)$, whose positive and negative energy parts are the annihilation-creation operators. ${ }^{5,8,9}$ It is the Hamiltonian and the annihilation-creation operators that form the dynamical symmetry algebra of the system, ${ }^{8,9}$ not the closure or dual-closure relations, nor the Askey-Wilson algebra relations. The $q$-oscillator algebra of Ref. 23 is the typical example of the dynamical symmetry algebra thus obtained.

The next is the difference in character of the Askey-Wilson algebra itself for the two cases; the pure imaginary shifts and the real shifts cases. The main scene of application of the AskeyWilson algebra is the theory of the orthogonal polynomials of a discrete variable. The $(q-)$ Racah polynomials are the typical example of this group. ${ }^{13,8}$ In our language, it is the theory of the eigenpolynomials of $\widetilde{\mathcal{H}}$ in discrete quantum mechanics with real shifts. One outstanding feature of these polynomials is the duality. ${ }^{8,21}$ For the eigenpolynomials of $\tilde{\mathcal{H}}$,

$$
\widetilde{\mathcal{H}} P_{n}(\eta(x))=\mathcal{E}(n) P_{n}(\eta(x)), \quad n=0,1, \ldots,
$$

there exist the dual polynomials $Q_{x}(\mathcal{E}(n))$, satisfying the relation

$$
P_{n}(\eta(x))=Q_{x}(\mathcal{E}(n)), \quad x=0,1, \ldots, \quad n=0,1, \ldots
$$

This duality $x \leftrightarrow n, \eta \sim \eta(x) \leftrightarrow \mathcal{E}(n) \sim \mathcal{H}$ is reflected in the symmetry between the pair of operators (called the Leonard pair $^{24}$ ) $K_{1}$ and $K_{2}$ or $A$ and $A^{\times}$in the Askey-Wilson algebra. The Askey- 
Wilson algebra or the closure and dual-closure relations are quite instrumental in clarifying various properties of the pair of orthogonal polynomials of a discrete variable. ${ }^{8,21}$

Now let us consider the discrete quantum mechanics with the pure imaginary shifts. In this case, the sinusoidal coordinate $\eta(x)$ takes the continuous value (spectrum) for the continuous range of $x \in\left(x_{1}, x_{2}\right)(2.2)$, which is markedly different from the spectrum of $\mathcal{H}$ postulated to take semi-infinite discrete values (2.6). The eigenpolynomials $\tilde{\mathcal{H}} P_{n}(\eta(x))=\mathcal{E}(n) P_{n}(\eta(x))$ depend on the continuous parameter $x$ and they have no dual polynomials. The Askey-Wilson and the Wilson polynomials are the typical examples. ${ }^{13,9}$ As shown in previous work ${ }^{5,9}$ and in Sec. III B, the essential information on exact solvability is contained only in closure relation (3.3). There is no evidence that the dual-closure relation plays a comparable role to the closure relation. Therefore, we may conclude that the apparent symmetry between $\mathcal{H}$ and $\eta$ or $K_{1}$ and $K_{2}$ or $A$ and $A^{\times}$in the Askey-Wilson algebra is quite misleading for the pure imaginary shifts case. In other words, a part of the Askey-Wilson algebra is irrelevant to the orthogonal polynomials of a continuous variable.

\section{Shape invariance}

Let us briefly review the condition and the outcome of the shape invariance ${ }^{1}$ in our language. In many cases the Hamiltonian contains some parameter(s), $\boldsymbol{\lambda}=\left(\lambda_{1}, \lambda_{2}, \ldots\right)$. Here we write parameter dependence explicitly, $\mathcal{H}(\boldsymbol{\lambda}), \mathcal{A}(\boldsymbol{\lambda}), \mathcal{E}(n ; \boldsymbol{\lambda}), \phi_{n}(x ; \boldsymbol{\lambda})$, etc., since it is the central issue. The shape invariance condition is ${ }^{6-9}$

$$
\mathcal{A}(\boldsymbol{\lambda}) \mathcal{A}(\boldsymbol{\lambda})^{\dagger}=\kappa \mathcal{A}\left(\boldsymbol{\lambda}^{\prime}\right)^{\dagger} \mathcal{A}\left(\boldsymbol{\lambda}^{\prime}\right)+\mathcal{E}(1 ; \boldsymbol{\lambda}),
$$

where $\kappa$ is a real positive parameter and $\boldsymbol{\lambda}^{\prime}$ is uniquely determined by $\boldsymbol{\lambda}$. Let us write the mapping as a function, $\boldsymbol{\lambda}^{\prime}=\operatorname{si}(\boldsymbol{\lambda})$. In concrete examples, if we take $\boldsymbol{\lambda}$ appropriately, $\boldsymbol{\lambda}^{\prime}$ has a simple additive form $\boldsymbol{\lambda}^{\prime}=\boldsymbol{\lambda}+\boldsymbol{\delta}$. The energy spectrum and the excited state wave function are determined by the data of the ground state wave function $\phi_{0}(x ; \boldsymbol{\lambda})$ and the energy of the first excited state $\mathcal{E}(1 ; \boldsymbol{\lambda})$ as follows:

$$
\begin{gathered}
\mathcal{E}(n ; \boldsymbol{\lambda})=\sum_{s=0}^{n-1} \kappa^{s} \mathcal{E}\left(1 ; \boldsymbol{\lambda}^{[s]}\right), \\
\phi_{n}(x ; \boldsymbol{\lambda}) \propto \mathcal{A}\left(\boldsymbol{\lambda}^{[0]}\right)^{\dagger} \mathcal{A}\left(\boldsymbol{\lambda}^{[1]}\right)^{\dagger} \mathcal{A}\left(\boldsymbol{\lambda}^{[2]}\right)^{\dagger} \cdots \mathcal{A}\left(\boldsymbol{\lambda}^{[n-1]}\right)^{\dagger} \phi_{0}\left(x ; \boldsymbol{\lambda}^{[n]}\right) .
\end{gathered}
$$

Here $\boldsymbol{\lambda}^{[n]}$ is $\boldsymbol{\lambda}^{[0]}=\boldsymbol{\lambda}, \boldsymbol{\lambda}^{[n]}=\operatorname{si}\left(\boldsymbol{\lambda}^{[n-1]}\right)(n=1,2, \ldots)$.

\section{Pure imaginary shifts case}

Here is a unified proof of the shape invariance for the discrete quantum mechanics with pure imaginary shifts. Shape invariance condition (3.45) is decomposed to the following set of two equations:

$$
\begin{gathered}
V\left(x-i \frac{\gamma}{2} ; \boldsymbol{\lambda}\right) V^{*}\left(x-i \frac{\gamma}{2} ; \boldsymbol{\lambda}\right)=\kappa^{2} V\left(x ; \boldsymbol{\lambda}^{\prime}\right) V^{*}\left(x-i \gamma ; \boldsymbol{\lambda}^{\prime}\right), \\
V\left(x+i \frac{\gamma}{2} ; \boldsymbol{\lambda}\right)+V^{*}\left(x-i \frac{\gamma}{2} ; \boldsymbol{\lambda}\right)=\kappa\left(V\left(x ; \boldsymbol{\lambda}^{\prime}\right)+V^{*}\left(x ; \boldsymbol{\lambda}^{\prime}\right)\right)-\mathcal{E}(1 ; \boldsymbol{\lambda}) .
\end{gathered}
$$

We assume that $\eta(x)$ satisfies the relation

$$
\eta(x)=\left[\frac{1}{2}\right]\left(\eta\left(x-i \frac{\gamma}{2}\right)+\eta\left(x+i \frac{\gamma}{2}\right)-\eta\left(-i \frac{\gamma}{2}\right)-\eta\left(i \frac{\gamma}{2}\right)\right) .
$$

Moreover, we assume that $\eta\left(x ; \boldsymbol{\lambda}^{\prime}\right)=\eta(x ; \boldsymbol{\lambda})$, for example, it is satisfied if $\eta(x)$ is $\boldsymbol{\lambda}$-independent. Both are easily verified in each of the explicit examples listed in Appendix A, (A1)-(A8). When 
the forms of potential functions (2.30) and (2.31) (with $L=2$ ) are substituted, shape invariance conditions (3.48) and (3.49) are satisfied. If we take $\left\{v_{k, 0}(k=0,1,2), v_{k, 1}(k=0,1)\right\}$ as $\boldsymbol{\lambda}$, then $\boldsymbol{\lambda}^{\prime}$ and $\mathcal{E}(1 ; \boldsymbol{\lambda})$ are

$$
\begin{aligned}
& \kappa v_{2,0}^{\prime}=-v_{1,1}, \\
& \kappa v_{1,1}^{\prime}=v_{2,0}+[2] v_{1,1} \text {, } \\
& \kappa v_{1,0}^{\prime}=\left[\frac{1}{2}\right]\left(v_{1,0}-v_{0,1}\right)+r_{-1}^{(2)}\left(\frac{\left[\frac{1}{4}\right]^{2}}{\left[\frac{1}{2}\right]^{2}} v_{2,0}+v_{1,1}\right), \\
& \kappa v_{0,1}^{\prime}=\left[\frac{1}{2}\right] v_{1,0}+\left[\frac{3}{2}\right] v_{0,1}+r_{-1}^{(2)}\left(\frac{\left[\frac{1}{4}\right]^{2}}{\left[\frac{1}{2}\right]^{2}} v_{2,0}+\frac{\left[\frac{1}{4}\right]\left[\frac{3}{4}\right]}{\left[\frac{1}{2}\right]^{2}} v_{1,1}\right), \\
& \kappa v_{0,0}^{\prime}=v_{0,0}+r_{-1}^{(2)}\left(\frac{\left[\frac{1}{4}\right]\left[\frac{3}{4}\right]}{\left[\frac{1}{2}\right]} v_{0,1}-\frac{\left[\frac{1}{4}\right]^{2}}{\left[\frac{1}{2}\right]^{2}} v_{1,0}\right)+\left[\frac{1}{2}\right]^{2}\left(\frac{\left[\frac{1}{4}\right]^{4}}{\left[\frac{1}{2}\right]^{4}} r_{-1}^{(2) 2}-\eta(-i \gamma) \eta(i \gamma)\right) v_{2,0} \\
& -\left[\frac{1}{2}\right]\left(\frac{\left[\frac{1}{4}\right]^{3}\left[\frac{3}{4}\right]}{\left[\frac{1}{2}\right]^{3}} r_{-1}^{(2) 2}+\left[\frac{3}{2}\right] \eta(-i \gamma) \eta(i \gamma)\right) v_{1,1} \\
& \mathcal{E}(1 ; \boldsymbol{\lambda})=v_{1,1} .
\end{aligned}
$$

Note that the above formula $\mathcal{E}(1 ; \boldsymbol{\lambda})$ is consistent with general formula (3.1). It is elementary to verify that the quadratic recursion formula generated by (3.51) and (3.52) coupled with shape invariance energy formulas (3.46) and (3.56) reproduces energy eigenvalue formula (3.1). However, the other formulas for parameter shifts (3.53)-(3.55) seem too complicated to be practical. As shown in Refs. 6, 7, 5, and 9, the parameter shifts are much simpler for the known examples.

Remark: In ordinary quantum mechanics there is a method for constructing a family of isospectral Hamiltonians, known as Crum's theorem. ${ }^{2}$ Recently we have obtained its discrete quantum mechanics version, see Ref. 25. If $\phi_{1}(x)$ takes a form $\phi_{1}(x)=\phi_{0}(x)($ const + const $\cdot \eta(x))$, which occurs indeed in the setting of this paper, the potential function of the first associated Hamiltonian is given by

$$
V^{[1]}\left(x+i \frac{\gamma}{2}\right)=V(x) \frac{\eta(x-i \gamma)-\eta(x)}{\eta(x)-\eta(x+i \gamma)} .
$$

Therefore, if shape invariance holds, $V(x)$ satisfies

$$
V\left(x+i \frac{\gamma}{2} ; \boldsymbol{\lambda}^{\prime}\right)=\kappa^{-1} V(x ; \boldsymbol{\lambda}) \frac{\eta(x-i \gamma ; \boldsymbol{\lambda})-\eta(x ; \boldsymbol{\lambda})}{\eta(x ; \boldsymbol{\lambda})-\eta(x+i \gamma ; \boldsymbol{\lambda})},
$$

in which the sinusoidal coordinate may depend on $\boldsymbol{\lambda}$. 


\section{Real shifts case}

Shape invariance (3.45) is equivalent to the following set of two equations:

$$
\begin{gathered}
B(x+1 ; \boldsymbol{\lambda}) D(x+1 ; \boldsymbol{\lambda})=\kappa^{2} B\left(x ; \boldsymbol{\lambda}^{\prime}\right) D\left(x+1 ; \boldsymbol{\lambda}^{\prime}\right), \\
B(x ; \boldsymbol{\lambda})+D(x+1 ; \boldsymbol{\lambda})=\kappa\left(B\left(x ; \boldsymbol{\lambda}^{\prime}\right)+D\left(x ; \boldsymbol{\lambda}^{\prime}\right)\right)+\mathcal{E}(1 ; \boldsymbol{\lambda}) .
\end{gathered}
$$

For the classified five types of $\eta(x),(\mathrm{i})^{\prime}-(\mathrm{v})^{\prime}$ in (A9)-(A13), the shape invariance holds. The boundary condition $D(0)=0(2.9)$ forces to choose $v_{0,0}$ as $v_{0,0}=-v_{0,1} \eta(-1)$. Thus, we take the parameters $\left\{v_{k, 0}(k=1,2), v_{k, 1}(k=0,1)\right\}$ [and $d$ for (ii)' and $\left.(\mathrm{v})^{\prime}\right]$ as $\boldsymbol{\lambda}$, then $\boldsymbol{\lambda}^{\prime}$ and $\mathcal{E}(1 ; \boldsymbol{\lambda})$ are

$$
\begin{aligned}
\kappa v_{2,0}^{\prime} & =-v_{1,1} \\
\kappa v_{1,1}^{\prime} & =v_{2,0}+[2] v_{1,1} \\
\kappa v_{1,0}^{\prime} & =\mu\left[\frac{1}{2}\right]\left(v_{1,0}-v_{0,1}\right)+\mu\left[\frac{1}{2}\right] \eta(1) v_{2,0}+\nu r_{-1}^{[2]} v_{1,1}, \\
\kappa v_{0,1}^{\prime} & =\mu\left(\left[\frac{1}{2}\right] v_{1,0}+\left[\frac{3}{2}\right] v_{0,1}\right)+\mu\left[\frac{1}{2}\right] \eta(1) v_{2,0}+\left(\nu r_{-1}^{[2]}+\mu\left[\frac{1}{2}\right](\eta(1)-\eta(-1))\right) v_{1,1}, \\
\mathcal{E}(1 ; \boldsymbol{\lambda}) & =-v_{1,1}, \\
d^{\prime} & = \begin{cases}d+1 & \text { for }(\mathrm{ii})^{\prime} \\
d q & \text { for }(\mathrm{v})^{\prime}\end{cases}
\end{aligned}
$$

in which $\mu$ and $\nu$ are constants,

$$
\mu=\left\{\begin{array}{ll}
1 & \text { for }(\mathrm{i})^{\prime}-(\mathrm{ii})^{\prime} \\
q^{-1 / 2} & \text { for }(\mathrm{iii})^{\prime} \\
q^{1 / 2} & \text { for }(\mathrm{iv})^{\prime}-(\mathrm{v})^{\prime},
\end{array} \quad \nu= \begin{cases}1 & \text { for }(\mathrm{i})^{\prime}-(\mathrm{iv})^{\prime} \\
\frac{1+d q}{1+d} & \text { for }(\mathrm{v})^{\prime} .\end{cases}\right.
$$

Quadratic recursion formulas (3.61) and (3.62) are exactly the same as those of pure imaginary shift case (3.51) and (3.52). Therefore, shape invariance energy formulas (3.46) and (3.65) produce same energy spectra (3.1). For the finite dimensional case, the natural number $N$ satisfying $B(N ; \boldsymbol{\lambda})=0(2.9)$ is also counted as a varying parameter. Then the shape invariance including the conditions

$$
N^{\prime}=N-1, \quad B\left(N^{\prime} ; \boldsymbol{\lambda}^{\prime}\right)=0
$$

is satisfied. As shown in Ref. 8, the parameter shifts are much simpler for the known examples.

\section{QUASIEXACTLY SOLVABLE $\tilde{\mathcal{H}}^{\prime}$}

QES means that only a finite part of the spectrum and the corresponding eigenfunctions can be obtained exactly. ${ }^{15}$ Usually such a theory contains a finite dimensional vector space ${ }^{26}$ consisting of polynomials of a certain degree which forms an invariant subspace of the "Hamiltonian" $\widetilde{\mathcal{H}}$, or more precisely its modification $\widetilde{\mathcal{H}}^{\prime}$. There are many ways to accomplish QES. The method of this paper can be considered as a simple generalization of the one in Ref. 27. That is, to add nonsolvable higher order term(s) together with compensation term(s) to an exactly solvable theory. As is clear from the construction, the sinusoidal coordinate plays an essential role. 
For a given positive integer $M$, let us try to find a QES "Hamiltonian" $\widetilde{\mathcal{H}}$, or more precisely its modification $\tilde{\mathcal{H}}^{\prime}$, having an invariant polynomial space $\mathcal{V}_{M}$,

$$
\tilde{\mathcal{H}}^{\prime} \mathcal{V}_{M} \subseteq \mathcal{V}_{M}
$$

For $L \geq 3,(2.34)$ is

$$
\tilde{\mathcal{H}} \eta(x)^{n}=\sum_{m=0}^{L-3} \eta(x)^{n+L-2-m} \sum_{j=0}^{m} e_{m, j, n}+(\text { a polynomial of degree } n \text { in } \eta(x)) .
$$

So let us define $\tilde{\mathcal{H}}^{\prime}$ by adding compensation terms to $\tilde{\mathcal{H}}$ as

$$
\tilde{\mathcal{H}}^{\prime}=\tilde{\mathcal{H}}-\sum_{m=0}^{L-3} e_{m}(M) \eta(x)^{L-2-m}, \quad e_{m}(M)=\sum_{j=0}^{\operatorname{def} m} e_{m, j, M} .
$$

Then we have $\tilde{\mathcal{H}}^{\prime} \eta(x)^{M} \in \mathcal{V}_{M}$. For $1 \leq m^{\prime} \leq L-3$, we have

$$
\begin{aligned}
\tilde{\mathcal{H}}^{\prime} \eta(x)^{M-m^{\prime}}= & \sum_{m=0}^{L-m^{\prime}-3} \eta(x)^{M+L-m^{\prime}-2-m}\left(\sum_{j=0}^{m} e_{m, j, M-m^{\prime}}-e_{m}(M)\right) \\
& +(\text { a polynomial of degree } M \text { in } \eta(x)) .
\end{aligned}
$$

If we could choose $v_{k, l}$ to satisfy all these conditions,

$$
\sum_{j=0}^{m} e_{m, j, M-m^{\prime}}-e_{m}(M)=0 \quad\left(1 \leq m^{\prime} \leq L-3,0 \leq m \leq L-m^{\prime}-3\right),
$$

then we would obtain $\tilde{\mathcal{H}}^{\prime} \mathcal{V}_{M} \subseteq \mathcal{V}_{M}$.

\section{A. QES with $L=3$}

For the $L=3$ case, $\tilde{\mathcal{H}}^{\prime}$ is defined by adding one compensation term of degree of 1 ,

$$
\tilde{\mathcal{H}}^{\prime}=\tilde{\mathcal{H}}-e_{0}(M) \eta(x), \quad \stackrel{\operatorname{def}}{e_{0}(M)=e_{0,0, M},}
$$

and we have achieved the quasiexact solvability $\widetilde{\mathcal{H}}^{\prime} \mathcal{V}_{M} \subseteq \mathcal{V}_{M}$. The number of exactly determined eigenstates is $M+1=\operatorname{dim}\left(\mathcal{V}_{M}\right)$. In this case there is no extra conditions for $v_{k, l}$. The explicit form of $e_{0}(M)$ is

$$
e_{0}(M)=\varepsilon \frac{\left[\frac{M}{2}\right]}{\left[\frac{1}{2}\right]}\left(\left[\frac{M-1}{2}\right] v_{3,0}+\left[\frac{M+1}{2}\right] v_{2,1}\right) .
$$

This QES theory has two more parameters $v_{3,0}$ and $v_{2,1}$ on top of those in the original exactly solvable theory $(L=2)$. Most known examples of QES belong to this category but those in ordinary quantum mechanics have only one extra parameter.

\section{B. QES with $L=4$}

This type of QES theory is new. For $L=4$ case, $\widetilde{\mathcal{H}}^{\prime}$ is defined by adding a linear and a quadratic in $\eta(x)$ compensation terms to the Hamiltonian $\tilde{\mathcal{H}}$, 
083502-18

$$
\tilde{\mathcal{H}}^{\prime}=\widetilde{\mathcal{H}}-e_{0}(M) \eta(x)^{2}-e_{1}(M) \eta(x), \quad \begin{aligned}
& \text { def } \\
& e_{0}(M)=e_{0,0, M},
\end{aligned} \quad \begin{aligned}
& \text { def } \\
& e_{1}(M)=e_{1,0, M}+e_{1,1, M},
\end{aligned}
$$

and $\tilde{\mathcal{H}}^{\prime} \eta(x)^{M} \in \mathcal{V}_{M}$. By using (2.28) we have

$$
\begin{aligned}
\tilde{\mathcal{H}}^{\prime} \eta(x)^{M-1} & =\eta(x)^{M+1}\left(e_{0,0, M-1}-e_{0}(M)\right)+(\text { a polynomial of degree } M \text { in } \eta(x)) \\
& =-\varepsilon \eta(x)^{M+1}\left([M-1] v_{4,0}+[M] v_{3,1}\right)+(\text { a polynomial of degree } M \text { in } \eta(x)) .
\end{aligned}
$$

In order to eliminate the $\eta(x)^{M+1}$ term, we choose $v_{3,1}$ as

$$
v_{3,1}=-\frac{[M-1]}{[M]} v_{4,0}
$$

We have achieved the quasiexact solvability $\tilde{\mathcal{H}}^{\prime} V_{M} \subseteq V_{M}$. The explicit forms of $e_{0}(M)$ and $e_{1}(M)$ are

$$
\begin{aligned}
& e_{0}(M)=-\varepsilon \frac{[4]\left[\frac{M}{2}\right]\left[\frac{M-1}{2}\right]}{\left[\frac{1}{2}\right][M+3]} v_{4,0}, \\
& e_{1}(M)= \frac{\left.\left[\frac{M}{2}\right] /\left[\frac{M-1}{2}\right] v_{3,0}+\left[\frac{M+1}{2}\right] v_{2,1}\right)-\varepsilon r_{-1}^{(2)} v_{4,0}}{\left.\frac{1}{2}\right]\left(\left[\frac{M(M-1)\left(M^{2}+5 M+8\right)}{M+3}\right.\right.} \\
& \times {\left[\frac{\left.\frac{M}{2}\right]\left[\frac{M-1}{2}\right]}{2\left[\frac{1}{r_{1}^{(1)}\left[\frac{1}{2}\right][M+3]}(4]-2[3]+2\left[\frac{1}{2}\right]\left[\frac{[2 M+5]}{\left[\frac{2 M+5}{2}\right]}\right) \text { for } r_{1}^{(1)} \neq 0 .\right.} \text { for } r_{1}^{(1)}=0\right.}
\end{aligned}
$$

The theory has three more free parameters on top of those of the original exactly solvable theory $(L=2)$.

\section{Non-QES for $L \geq 5$}

The higher $L$ becomes, the number of conditions to be satisfied (4.5) increases more rapidly than the number of additional parameters. We will show that $L \geq 5$ case cannot be made QES. Condition (4.5) with $m=0$ gives $e_{0,0, M}=e_{0,0, M-m^{\prime}}\left(1 \leq m^{\prime} \leq L-3\right)$, and by using (2.37) and (2.28), we obtain

$$
\left[M-\frac{m^{\prime}+1}{2}\right] v_{L, 0}+\left[M-\frac{m^{\prime}-1}{2}\right] v_{L-1,1}=0 \quad\left(1 \leq m^{\prime} \leq L-3\right) .
$$

For $L \geq 5$ case, these equations do not have nontrivial solutions. For $m^{\prime}=1,2$ we obtain 


$$
\left(\begin{array}{cc}
{[M-1]} & {[M]} \\
{\left[M-\frac{3}{2}\right]} & {\left[M-\frac{1}{2}\right]}
\end{array}\right)\left(\begin{array}{c}
v_{L, 0} \\
v_{L-1,1}
\end{array}\right)=\left(\begin{array}{l}
0 \\
0
\end{array}\right) .
$$

The determinant of this matrix is $\left[\frac{1}{2}\right]$ which does not vanish. Thus, we obtain $v_{L, 0}=v_{L-1,1}=0$. Namely, there is no $v_{k, l}(k+l=L)$ term. Therefore, $L \geq 5$ case cannot be made QES.

\section{V. (QUASI-)EXACTLY SOLVABLE HAMILTONIAN}

If there exists a ground state wave function $\phi_{0}(x)$ which satisfies (2.14) (and $\left\|\phi_{0}\right\|<\infty$, the Hermiticity of $\mathcal{H}$ ), we can return to the Hamiltonian $\mathcal{H}$ from the "Hamiltonian" $\widetilde{\mathcal{H}}$ by inverse similarity transformation (2.15). In the same way the QES Hamiltonian $\mathcal{H}^{\prime}$ is obtained from $\widetilde{\mathcal{H}}^{\prime}$ by the inverse similarity transformation in terms of the pseudo-ground-state wave function $\phi_{0}(x)$ satisfying $\mathcal{A} \phi_{0}=0$ (2.14),

$$
\mathcal{H}^{\prime}=\phi_{0}(x) \circ \tilde{\mathcal{H}}^{\prime} \circ \phi_{0}(x)^{-1} .
$$

It should be noted that $\phi_{0}(x)$ is neither the ground state nor an eigenstate of the total Hamiltonian $\tilde{\mathcal{H}}^{\prime}$. Thus, it is called the pseudo-ground-state wave function. For the $L=3,4$ cases, we have

$$
\begin{gathered}
L=3: \mathcal{H}^{\prime}=\mathcal{H}-e_{0}(M) \eta(x), \\
{ }_{\text {def }}{ }_{4}: \mathcal{H}^{\prime}=\mathcal{H}-e_{0}(M) \eta(x)^{2}-e_{1}(M) \eta(x) .
\end{gathered}
$$

Let us note that the Hamiltonian $\mathcal{H}^{\prime}$ does not factorize and the semi positive-definite spectrum is lost due to the compensation terms. For the pure imaginary shifts case, the existence of (pseudo)groundstate wave function $\phi_{0}(x)$ strongly depends on the concrete form of $V(x)$ and its parameter range. There is no general formula to write down $\phi_{0}(x)$ in terms of $V(x)$. On the other hand, for the real shift case, the (pseudo-)ground state wave function $\phi_{0}(x)$ is uniquely given by ${ }^{8}$

$$
\phi_{0}(x)=\sqrt{\prod_{y=0}^{x-1} \frac{B(y)}{D(y+1)}} .
$$

The positivity of $B(x)$ and $D(x)$ (2.9) restricts their parameter range. For the infinite case $x$ $\in[0, \infty)$, the square summability $\left\|\phi_{0}\right\|<\infty$ restricts the asymptotic forms of $B(x)$ and $D(x)$.

\section{SUMMARY AND THE RECIPE}

Based on the sinusoidal coordinate $\eta(x)$, we have systematically explored a unified theory of one-dimensional exactly and quasiexactly solvable "discrete" quantum mechanical models. The Hamiltonians of discrete quantum mechanics have shift operators as exponentiated forms of the momentum operator $p=-i \partial_{x}, e^{ \pm \beta p}=e^{\mp i \beta \partial_{x}}$. This method applies to both the pure imaginary shifts $\left(\beta=\gamma \in \mathbb{R}_{\neq 0}\right)$ and the real shifts cases $(\beta=i=\sqrt{-1})$, which have a continuous and a discrete dynamical variable $x$, respectively. The main input is the special form of the potential functions $V_{ \pm}(x)(2.30)$ and (2.31), with which the "Hamiltonian" $\widetilde{\mathcal{H}}(2.16)$ maps a polynomial in $\eta(x)$ into another. We obtain exactly solvable models (degree $n \rightarrow n$ ) and quasiexactly solvable models (degree $n \rightarrow n+1, n+2)$ by adding compensation terms, which are linear and quadratic in $\eta(x)$, respectively. The QES Hamiltonians based on the mapping (degree $n \rightarrow n+2)$ are new.

The corresponding result in the ordinary QM can be found in Appendix of Ref. 5. This early work, however, does not cover the quasiexactly solvable cases. In this connection, see the recent developments. ${ }^{28}$ 
The present paper is for the presentation of the basic formalism. Application and concrete examples will be explored in a subsequent publication. ${ }^{14}$ The explicit forms of various sinusoidal coordinates are listed in Appendix A.

The forms of the (pseudo-)groundstate wave functions $\phi_{0}(x)$ for the pure imaginary shifts (the continuous variable) case depend on the choice of the sinusoidal coordinates. They are "gamma functions" having various shift properties; the (Euler) gamma function for (i) and (ii), (A1) and (A2), the $q$-gamma function (or the $q$-Pochhammer symbol) for (iii) and (iv), (A3) and (A4), the double gamma function (or the quantum dilogarithm function) for (v)-(viii), (A5)-(A8). In a subsequent publication we will present explicit examples of new Hamiltonians based on (A5)-(A8). Their eigenfunctions contain orthogonal polynomials and the double gamma functions as the orthogonality measure functions. Eigenpolynomials $P_{n}(\eta(x))$ for exactly solvable QM belong to the Askey-scheme of hypergeometric orthogonal polynomials. Various examples of exactly solvable QM were investigated for (i)-(iv) (A1)-(A4), (Ref. 9) and (i)' $-(\mathrm{v})^{\prime}$, (A9)-(A13), ${ }^{8}$ and quasiexactly solvable QMs were partially examined in Refs. 16 and 17.

The simple recipe to construct an exactly or quasiexactly solvable Hamiltonian is as follows.

(1) Choose the sinusoidal coordinate among (A1)-(A8) if the variable is continuous, among (A9)-(A13) for the discrete variable.

(2) Choose $L=2$ for exact solvability and $L=3,4$ for quasiexact solvability and write down the "Hamiltonian" $\tilde{\mathcal{H}}$ in polynomial spaces $(2.30)$ and (2.31) with the free parameters $v_{k, l}, k+l$ $\leq L, l=0,1$. For the quasiexactly solvable case, add proper compensation terms (4.6) and (4.7) for the $L=3$ case and (4.8)-(4.12) for the $L=4$ case.

(3) Determine the (pseudo-)ground state $\phi_{0}$ as a zero mode of $\mathcal{A},(2.14)$, which can be found among the various gamma functions listed as above or (5.4) for the discrete variable case.

(4) Restrict the parameter ranges so that the square-integrability of $\phi_{0}$ and the Hermiticity is satisfied for the continuous variable case. For the discrete variable case the positivity $B(x)$ $>0$ and $D(x)>0$ and the boundary condition(s) $D(0)=0,(B(N)=0)(2.9)$ are the conditions to restrict the parameters. For the infinite dimensional case the square summability $\sum_{x=0}^{\infty} \phi_{0}^{2}(x)<\infty$ must be satisfied, too.

(5) Apply inverse similarity transformation (5.1) in terms of $\phi_{0}$ on the "Hamiltonian" in the polynomial space to get the Hamiltonian $\mathcal{H}$ or $\mathcal{H}^{\prime}$.

\section{ACKNOWLEDGMENTS}

We thank Paul Terwilliger for fruitful discussion. This work is supported in part by Grantsin-Aid for Scientific Research from the Ministry of Education, Culture, Sports, Science and Technology, Grant Nos. 18340061 and 19540179.

\section{APPENDIX A: LIST OF SINUSOIDAL COORDINATES}

Here we list the explicit forms of the sinusoidal coordinates, based on which various concrete examples of exactly and quasiexactly solvable theories are constructed.

These are eight sinusoidal coordinates for the pure imaginary shifts case (continuous $x$ ),

$$
\begin{aligned}
& \text { (i): } \eta(x)=x, \quad-\infty<x<\infty, \quad \gamma=1, \\
& \text { (ii): } \eta(x)=x^{2}, \quad 0<x<\infty, \quad \gamma=1, \\
& \text { (iii): } \eta(x)=1-\cos x, \quad 0<x<\pi, \quad \gamma \in \mathbb{R}_{\neq 0}, \\
& \text { (iv): } \eta(x)=\sin x, \quad-\frac{\pi}{2}<x<\frac{\pi}{2}, \quad \gamma \in \mathbb{R}_{\neq 0},
\end{aligned}
$$




$$
\begin{aligned}
& \text { (v): } \eta(x)=1-e^{-x}, \quad-\infty<x<\infty, \quad \gamma \in \mathbb{R}_{\neq 0}, \\
& \text { (vi): } \eta(x)=e^{x}-1, \quad-\infty<x<\infty, \quad \gamma \in \mathbb{R}_{\neq 0}, \\
& \text { (vii): } \eta(x)=\cosh x-1, \quad 0<x<\infty, \quad \gamma \in \mathbb{R}_{\neq 0}, \\
& \text { (viii): } \eta(x)=\sinh x, \quad-\infty<x<\infty, \quad \gamma \in \mathbb{R}_{\neq 0},
\end{aligned}
$$

and five sinusoidal coordinates for the real shifts case (integer $x$ ),

(i) $)^{\prime}: \eta(x)=x$,

(ii) $)^{\prime} \eta(x)=\epsilon^{\prime} x(x+d), \quad \epsilon^{\prime}=\left\{\begin{array}{cc}1 & \text { for } d>-1 \\ -1 & \text { for } d<-N\end{array}\right.$

(iii)' ${ }^{\prime}: \eta(x)=1-q^{x}$,

$(\text { iv) })^{\prime}: \eta(x)=q^{-x}-1$,

$$
(\mathrm{v})^{\prime}: \eta(x)=\epsilon^{\prime}\left(q^{-x}-1\right)\left(1-d q^{x}\right), \quad \epsilon^{\prime}=\left\{\begin{array}{cc}
1 & \text { for } d<q^{-1} \\
-1 & \text { for } d>q^{-N}
\end{array}\right.
$$

where $0<q<1$. As shown in detail in Sec. 4C of Ref. 8, the above five sinusoidal coordinates for real shifts (A9)-(A13) exhaust all the solutions of (2.18)-(2.20) up to a multiplicative factor. On the other hand, those for the pure imaginary shifts (i)-(viii) (A1)-(A8) are merely typical examples satisfying all the postulates for sinusoidal coordinate (2.18)-(2.20) and the extra one used for shape invariance (3.50). It is easy to see that $\eta(x)=x+\sinh (2 \pi x),-\infty<x<\infty, \gamma=1$ is a good sinusoidal coordinate for the imaginary shifts but it fails to fulfill extra condition (3.50).

\section{APPENDIX B: HERMITICITY OF THE HAMILTONIAN}

In this appendix we recapitulate the proof of the Hermiticity of the Hamiltonian $\mathcal{H}$ (2.7) for the pure imaginary shift (continuous variable) case. ${ }^{16,9}$ For the real shifts (discrete variable) case the Hamiltonian $\mathcal{H}(2.7)$ is a Hermitian matrix (real symmetric matrix) and there is no problem for the Hermiticity. Thus, we consider only the continuous variables case, in which the wave functions and the potential functions are analytic function of $x$ as explained in Sec. II. The *-operation on analytic functions is also defined there.

By using the formula $(A B)^{\dagger}=B^{\dagger} A^{\dagger}$, we obtain $\mathcal{H}^{\dagger}=\mathcal{H}$ but this is formal Hermiticity. In order to demonstrate the true Hermiticity of $\mathcal{H}$, we have to show $(g, \mathcal{H} f)=(\mathcal{H} g, f)$ with respect to inner product (2.4). Since the eigenfunctions considered in this paper have the form $\phi_{0}(x) P_{n}(\eta(x))$, it is sufficient to check for $f(x)=\phi_{0}(x) P(\eta(x))$ and $g(x)=\phi_{0}(x) Q(\eta(x))$, where $P(\eta)$ and $Q(\eta)$ are polynomials in $\eta$. Since $\mathcal{H}$ is real $\mathcal{H}^{*}=\mathcal{H}$, namely, $\mathcal{H}$ maps a "real" function to a "real" function $\left(f^{*}(x)=f(x) \Rightarrow(\mathcal{H} f)^{*}=(\mathcal{H} f)\right)$, we can take $\phi_{0}(x), P(\eta(x))$, and $Q(\eta(x))$ to be "real" functions of $x$ and we do so in the following.

The (pseudo-)ground state wave function $\phi_{0}(x)$ is determined as a zero mode of $\mathcal{A}, \mathcal{A} \phi_{0}=0$ (2.14). The equation reads

$$
\sqrt{V^{*}\left(x-i \frac{\gamma}{2}\right)} \phi_{0}\left(x-i \frac{\gamma}{2}\right)=\sqrt{V\left(x+i \frac{\gamma}{2}\right)} \phi_{0}\left(x+i \frac{\gamma}{2}\right) .
$$

Let us define $T_{+}=\sqrt{V(x)} e^{\gamma p} \sqrt{V^{*}(x)}$ and $T_{-}=\sqrt{V^{*}(x)} e^{-\gamma p} \sqrt{V(x)}$. Then Hamiltonian (2.7) is $\mathcal{H}=T_{+}$ $+T_{-}-V(x)-V^{*}(x)$. For the QES case, the compensation terms are added. It is obvious that the 
function part $-V(x)-V^{*}(x)$ (plus possible compensation terms) is Hermitian by itself. Let us define two analytic functions $F(x)$ and $G(x)$ as follows:

$$
\begin{aligned}
& g^{*}(x) T_{+} f(x)=\phi_{0}^{*}(x) Q^{*}\left(\eta^{*}(x)\right) \sqrt{V(x)} \sqrt{V^{*}(x-i \gamma)} \phi_{0}(x-i \gamma) P(\eta(x-i \gamma))=F(x), \\
& g^{*}(x) T_{-} f(x)=\phi_{0}^{*}(x) Q^{*}\left(\eta^{*}(x)\right) \sqrt{V^{*}(x)} \sqrt{V(x+i \gamma)} \phi_{0}(x+i \gamma) P(\eta(x+i \gamma))=G(x) .
\end{aligned}
$$

Then we have

$$
\begin{aligned}
& \left(T_{+} g\right)^{*}(x) f(x)=\phi_{0}^{*}(x+i \gamma) Q^{*}\left(\eta^{*}(x+i \gamma)\right) \sqrt{V^{*}(x)} \sqrt{V(x+i \gamma)} \phi_{0}(x) P(\eta(x))=F(x+i \gamma), \\
& \left(T_{-} g\right)^{*}(x) f(x)=\phi_{0}^{*}(x-i \gamma) Q^{*}\left(\eta^{*}(x-i \gamma)\right) \sqrt{V(x)} \sqrt{V^{*}(x-i \gamma)} \phi_{0}(x) P(\eta(x))=G(x-i \gamma) .
\end{aligned}
$$

By using (B1) and the "reality" of $\phi_{0}(x), \eta(x), P(\eta), Q(\eta)$, we obtain

$$
\begin{gathered}
g^{*}(x) T_{+} f(x)=V(x) \phi_{0}(x)^{2} Q(\eta(x)) P(\eta(x-i \gamma))=F(x), \\
g^{*}(x) T_{-} f(x)=V^{*}(x) \phi_{0}(x)^{2} Q(\eta(x)) P(\eta(x+i \gamma))=G(x), \\
\left(T_{+} g\right)^{*}(x) f(x)=V(x+i \gamma) \phi_{0}(x+i \gamma)^{2} Q(\eta(x+i \gamma)) P(\eta(x))=F(x+i \gamma), \\
\left(T_{-} g\right)^{*}(x) f(x)=V^{*}(x-i \gamma) \phi_{0}(x-i \gamma)^{2} Q(\eta(x-i \gamma)) P(\eta(x))=G(x-i \gamma) .
\end{gathered}
$$

Therefore, the necessary and sufficient condition for the Hermiticity of the Hamiltonian becomes

$$
\int_{x_{1}}^{x_{2}}(F(x)+G(x)) d x=\int_{x_{1}}^{x_{2}}(F(x+i \gamma)+G(x-i \gamma)) d x .
$$

Of course it is required that there is no singularity on the integration contours.

Let $C_{ \pm}$be the rectangular contours $x_{1} \rightarrow x_{2} \rightarrow x_{2} \pm i \gamma \rightarrow x_{1} \pm i \gamma \rightarrow x_{1}$ and $D_{ \pm}$be the regions surrounded by $C_{ \pm}$including the contours. Under the assumption that $F(x)$ and $G(x)$ do not have singularities on $C_{+}$and $C_{-}$, respectively, the residue theorem implies that (B10) is rewritten as

$$
\begin{gathered}
\int_{0}^{\gamma}\left(F\left(x_{2}+i y\right)-F\left(x_{1}+i y\right)-G\left(x_{2}-i y\right)+G\left(x_{1}-i y\right)\right) d y \\
\quad=2 \pi \frac{\gamma}{|\gamma|}\left(\sum_{x: \text { pole in } D_{+}} \operatorname{Res}_{x} F(x)-\sum_{x \text { :pole in } D_{-}} \operatorname{Res}_{x} G(x)\right) .
\end{gathered}
$$

If there are singularities on the contours $x_{2} \rightarrow x_{2} \pm i \gamma$ or $x_{1} \pm i \gamma \rightarrow x_{1}$, we deform the contours and redefine $C_{ \pm}$and $D_{ \pm}$in order to avoid singularities on $C_{ \pm}$. For simplicity we have assumed no singularity on $C_{ \pm}$in the text.

We will mention several sufficient conditions for (B11). If $F(x)$ and $G(x)$ are holomorphic in $D_{+}$and $D_{-}$, respectively, the right hand side of (B11) vanishes. In the following we assume this.

Case 1: $x_{1}=-\infty, x_{2}=\infty$.

If $\phi_{0}(x)$ is rapidly decreasing [e.g., exponential in $\eta(x)$ ] at $x \sim \pm \infty$, then (B11) is satisfied.

Case 2: $x_{1}=0, x_{2}=\infty$.

If $\phi_{0}(x)$ is rapidly decreasing [e.g., exponential in $\eta(x)$ ] at $x \sim \infty$, then (B11) becomes $\int_{0}^{\gamma}(F(i y)-G(-i y)) d y=0$. This is satisfied if $F(i y)=G(-i y), y \in(0, \gamma)$. As a sufficient condition for $F(i y)=G(-i y)$, we give the following three reflection properties:

$$
\phi_{0}(-x)=\phi_{0}(x), \quad \eta(-x)=\eta(x), \quad V^{*}(x)=V(-x)
$$


Case 3: $x_{2}=x_{1}+\omega(0<\omega<\infty)$.

Condition (B11) becomes $\int_{0}^{\gamma}\left(F\left(x_{1}+\omega+i y\right)-F\left(x_{1}+i y\right)-G\left(x_{1}+\omega-i y\right)+G\left(x_{1}-i y\right)\right) d y=0$. This is satisfied if $F\left(x_{1}+\omega+i y\right)=G\left(x_{1}+\omega-i y\right)$ and $F\left(x_{1}+i y\right)=G\left(x_{1}-i y\right), y \in(0, \gamma)$. As a sufficient condition for $F\left(x_{1}+\omega+i y\right)=G\left(x_{1}+\omega-i y\right)$ and $F\left(x_{1}+i y\right)=G\left(x_{1}-i y\right)$, we give the following reflection relations and the periodicity:

$$
\begin{gathered}
\phi_{0}\left(-x+x_{1}\right)=\phi_{0}\left(x+x_{1}\right), \quad \eta\left(-x+x_{1}\right)=\eta\left(x+x_{1}\right), \quad V^{*}\left(x+x_{1}\right)=V\left(-x+x_{1}\right), \\
\phi_{0}(x+2 \omega)=\phi_{0}(x), \quad \eta(x+2 \omega)=\eta(x), \quad V(x+2 \omega)=V(x) .
\end{gathered}
$$

\section{APPENDIX C: EIGENVALUES AND EIGENVECTORS FOR AN UPPER-TRIANGULAR MATRIX}

Let $A=\left(a_{i j}\right)_{1 \leq i, j \leq n}$ be an upper-triangular matrix, namely, $a_{i j}=0$ for $i>j$. Its eigenvalues are $\alpha_{i}=a_{i i}(i=1, \ldots, n)$. When $\alpha_{i}$ 's are mutually distinct, the eigenvector corresponding to the eigenvalue $\alpha_{i}$ is given by the determinant

$$
\left|\begin{array}{lllll}
\boldsymbol{e}_{1} & \boldsymbol{e}_{2} & \boldsymbol{e}_{3} & \cdots & \boldsymbol{e}_{i} \\
\alpha_{1}-\alpha_{i} & a_{12} & a_{13} & \cdots & a_{1 i} \\
& \alpha_{2}-\alpha_{i} & a_{23} & \cdots & a_{2 i} \\
& & \ddots & \ddots & \vdots \\
0 & & & \alpha_{i-1}-\alpha_{i} & a_{i-1 i}
\end{array}\right|,
$$

where $\left\{\boldsymbol{e}_{i}\right\}$ is the natural basis, $\left(\boldsymbol{e}_{i}\right)_{j}=\delta_{i j}$.

${ }^{1}$ L. E. Gendenshtein, JETP Lett. 38, 356 (1983).

${ }^{2}$ M. M. Crum, Quart. J. Math. Oxford Ser. (2) 6, 121 (1955); e-print arXiv:physics/9908019.

${ }^{3}$ L. Infeld and T. E. Hull, Rev. Mod. Phys. 23, 21 (1951).

${ }^{4}$ See, for example, a review: F. Cooper, A. Khare, and U. Sukhatme, Phys. Rep. 251, 267 (1995).

${ }^{5}$ S. Odake and R. Sasaki, J. Math. Phys. 47, 102102 (2006); e-print arXiv:quant-ph/0605215; Phys. Lett. B 641, 112 (2006); e-print arXiv:quant-ph/0605221

${ }^{6}$ S. Odake and R. Sasaki, J. Nonlinear Math. Phys. 12, Suppl. 1, 507 (2005); e-print arXiv:hep-th/0410102.

${ }^{7}$ S. Odake and R. Sasaki, J. Math. Phys. 46, 063513 (2005); e-print arXiv:hep-th/0410109.

${ }^{8}$ S. Odake and R. Sasaki, J. Math. Phys. 49, 053503 (2008); e-print arXiv:0712.4106 [math.CA].

${ }^{9}$ S. Odake and R. Sasaki, Prog. Theor. Phys. 119, 663 (2008); e-print arXiv:0802.1075 [quant-ph].

${ }^{10}$ S. Odake and R. Sasaki, Prog. Theor. Phys. 114, 1245 (2005); e-print arXiv:hep-th/0512155.

${ }^{11}$ G. E. Andrews, R. Askey, and R. Roy, Special Functions, Encyclopedia of Mathematics and Its Applications (Cambridge University Press, Cambridge) (1999).

${ }^{12}$ M. E. H. Ismail, Classical and Quantum Orthogonal Polynomials in One Variable, Encyclopedia of Mathematics and Its Applications (Cambridge University Press, Cambridge) (2005).

${ }^{13}$ R. Koekoek and R. F. Swarttouw, e-print arXiv:math.CA/9602214.

${ }^{14} \mathrm{~S}$. Odake and R. Sasaki (unpublished).

${ }^{15}$ See, for example, A. G. Ushveridze, Sov. Phys. Lebedev. Inst. Rep. 2, 50 (1988); A. G. Ushveridze, Quasi-exactly solvable models in quantum mechanics (IOP, Bristol, 1994); A. Y Morozov, A. M. Perelomov, A. A. Rosly, M. A. Shifman, and A. V. Turbiner, Int. J. Mod. Phys. A 5, 803 (1990).

${ }^{16}$ R. Sasaki, J. Math. Phys. 48, 122104 (2007); e-print arXiv:0708.0702 [nlin.SI]; S. Odake and R. Sasaki, J. Math. Phys. 48, 122105 (2007); e-print arXiv:0708.0716 [nlin.SI].

${ }^{17}$ R. Sasaki, J. Nonlinear Math. Phys. 15, Supp. 3, 373 (2008); e-print arXiv:0712.2616 [nlin.SI].

${ }^{18}$ A. S. Zhedanov, Theor. Math. Phys. 89, 1146 (1991).

${ }^{19}$ Ya. I. Granovskii, I. M. Lutzenko, and A. Zhedanov, Ann. Phys. 217, 1 (1992).

${ }^{20}$ L. Vinet and A. Zhedanov, Symmetry, Integr. Geom.: Methods Appl. 4, 015 (2008); e-print arXiv:0802.0744 [math.QA].

${ }^{21}$ P. Terwilliger, Lecture Notes For The Summer School On Orthogonal Polynomials And Special Functions, Universidad Carlos III de Madrid, Leganes, Spain, 8 July-18 July 2004; e-print arXiv:math.QA/0408390.

${ }^{22}$ T. H. Koornwinder, Symmetry, Integr. Geom.: Methods Appl. 4, 052 (2008); e-print arXiv:0711.2320 [math.QA].

${ }^{23}$ S. Odake and R. Sasaki, Phys. Lett. B 663, 141 (2008); e-print arXiv:0710.2209 [hep-th].

${ }^{24}$ D. Leonard, SIAM J. Math. Anal. 13, 656 (1982).

${ }^{25}$ S. Odake and R. Sasaki, Prog. Theor. Phys. 122, 1067 (2009); e-print arXiv:0902.2593 [math-ph].

${ }^{26}$ A. V. Turbiner, Commun. Math. Phys. 118, 467 (1988). 
${ }^{27}$ R. Sasaki and K. Takasaki, J. Phys. A 34, 9533 (2001); R. Sasaki and K. Takasaki, J. Phys. A 34, 10335 (2001); e-print arXiv:hep-th/0109008.

${ }^{28}$ C.-L. Ho, Ann. Phys. 323, 2241 (2008); e-print arXiv:0801.0944 [hep-th]; C.-L. Ho, Ann. Phys. 324, 1095 (2009); e-print arXiv:0809.5253 [quant-ph]. 